\title{
Expression regulation of multiple key genes to improve L-threonine in Escherichia coli
}

\author{
Lei Zhao ${ }^{1,2}$, Ying Lu ${ }^{3}$, Jun Yang ${ }^{1,2}$, Yu Fang ${ }^{1,2}$, Lifei Zhu ${ }^{1,2}$, Zhixiang Ding ${ }^{1,2}$, Chenhui Wang ${ }^{1,2}$, Wenjian Ma ${ }^{1,2}$, \\ Xiaoqing $\mathrm{Hu}^{1,2}$ and Xiaoyuan Wang ${ }^{1,2,4^{*}}$ (1)
}

\begin{abstract}
Background: Escherichia coli is an important strain for L-threonine production. Genetic switch is a ubiquitous regulatory tool for gene expression in prokaryotic cells. To sense and regulate intracellular or extracellular chemicals, bacteria evolve a variety of transcription factors. The key enzymes required for L-threonine biosynthesis in E. coli are encoded by the thr operon. The thr operon could coordinate expression of these genes when L-threonine is in short supply in the cell.
\end{abstract}

Results: The thrL leader regulatory elements were applied to regulate the expression of genes $i c \mid R$, $\operatorname{arcA}, c p x R$, gadE, fadR and pykF, while the threonine-activating promoters $P_{\text {cysht }} P_{c y s}$ and $P_{\text {cys } D}$ were applied to regulate the expression of gene $a s p C$, resulting in the increase of L-threonine production in an L-threonine producing E. coli strain TWF001. Firstly, different parts of the regulator thrL were inserted in the icIR regulator region in TWF001, and the best resulting strain TWF063 produced $16.34 \mathrm{~g}$ L-threonine from $40 \mathrm{~g}$ glucose after $30 \mathrm{~h}$ cultivation. Secondly, the gene aspC following different threonine-activating promoters was inserted into the chromosome of TWF063, and the best resulting strain TWF066 produced $17.56 \mathrm{~g}$ L-threonine from $40 \mathrm{~g}$ glucose after $30 \mathrm{~h}$ cultivation. Thirdly, the effect of expression regulation of $\operatorname{arcA}, c p x R, g a d E$, pykF and fadR was individually investigated on L-threonine production in TWF001. Finally, using TWF066 as the starting strain, the expression of genes arcA, $c p x R$, gadE, pykF and fadR was regulated individually or in combination to obtain the best strain for L-threonine production. The resulting strain TWF083, in which the expression of seven genes (iclR, aspC, arcA, cpxR, gadE, pykF, fadR and aspC) was regulated, produced $18.76 \mathrm{~g}$ L-threonine from $30 \mathrm{~g}$ glucose, $26.50 \mathrm{~g}$ L-threonine from $40 \mathrm{~g}$ glucose, or $26.93 \mathrm{~g}$ L-threonine from $50 \mathrm{~g}$ glucose after $30 \mathrm{~h}$ cultivation. In $48 \mathrm{~h}$ fed-batch fermentation, TWF083 could produce $116.62 \mathrm{~g} / \mathrm{L}$ L-threonine with a yield of $0.486 \mathrm{~g} / \mathrm{g}$ glucose and productivity of $2.43 \mathrm{~g} / \mathrm{L} / \mathrm{h}$.

Conclusion: The genetic engineering through the expression regulation of key genes is a better strategy than simple deletion of these genes to improve L-threonine production in E. coli. This strategy has little effect on the intracellular metabolism in the early stage of the growth but could increase L-threonine biosynthesis in the late stage.

Keywords: Escherichia coli, L-Threonine, Expression regulation, Metabolic flux

*Correspondence: xwang@jiangnan.edu.cn

1 State Key Laboratory of Food Science and Technology, Jiangnan University, 1800 Lihu Avenue, Wuxi 214122, China

Full list of author information is available at the end of the article

\section{Introduction}

L-Threonine has an increasing market demand in pharmaceutical, cosmetics and food industry [1]. Escherichia coli is an important strain for $\mathrm{L}$-threonine production [24]. Efforts have been made to improve the productivity of L-threonine in $E$. coli, such as increasing the carbon flux to $\mathrm{L}$-threonine $[5,6]$, enhancing the export of $\mathrm{L}$-threonine

(c) The Author(s) 2020. This article is licensed under a Creative Commons Attribution 4.0 International License, which permits use, sharing, adaptation, distribution and reproduction in any medium or format, as long as you give appropriate credit to the original author(s) and the source, provide a link to the Creative Commons licence, and indicate if changes were made. The images or other third party material in this article are included in the article's Creative Commons licence, unless indicated otherwise in a credit line to the material. If material is not included in the article's Creative Commons licence and your intended use is not permitted by statutory regulation or exceeds the permitted use, you will need to obtain permission directly from the copyright holder. To view a copy of this licence, visit http://creativeco mmons.org/licenses/by/4.0/. The Creative Commons Public Domain Dedication waiver (http://creativecommons.org/publicdomain/ zero/1.0/) applies to the data made available in this article, unless otherwise stated in a credit line to the data. 
[7], weakening the competitive pathway and reducing the consumption of L-threonine $[8,9]$.

Genetic switch is a ubiquitous regulatory tool for gene expression in prokaryotic cells [10]. In E. coli, L-threonine is synthesized through a series of reactions from L-aspartate, which is converted from oxaloacetate by aspartate aminotransferase encoded by aspC. The key enzymes required for L-threonine biosynthesis in $E$. coli are encoded by the $t h r$ operon. The $t h r$ operon could coordinate expression of these genes when L-threonine is in short supply in the cell. This regulation is controlled by the lead sequence of the $t h r$ operon $(t h r L)$. The $t h r L$ contains an efficient ribosome-binding site (RBS) and can form a twenty-one-amino-acid leader peptide. From the 6th codons of this leader peptide, it starts to encode L-threonine residues, therefore, it can regulate the transcription of the thr operon through the availability of L-threonine, by forming different base-paired RNA structure [11]. Since thrL inhibits L-threonine accumulation it has been mutated in L-threonine producing E. coli, such as TWF001 [9]. On the other hand, some biosynthetic pathways in E. coli can be activated in the presence of high concentration of L-threonine, such as the sulfate metabolism branch of the cysteine biosynthetic pathway which includes the genes $c y s D N, c y s I I$ and $c y s H$ [12].

To sense and regulate intracellular or extracellular chemicals, bacteria evolve a variety of transcription factors. Based on this principle, various biosensors for amino acids and their precursors, including L-methionine, L-leucine, L-isoleucine, L-valine [13, 14], L-lysine, L-arginine, L-serine, $O$-acetyl-L-serine [15], $O$-acetyl homoserine [16], and oxygen [17] have been constructed, and some of these sensors have been successfully applied to the high-throughput screening [12, 18 , 19] and systems metabolic engineering [20]. L-Threonine production in $E$. coli can be enhanced by regulating IclR $[8,9]$, a transcription factor. IclR is a repressor for the aceBAK operon (Fig. 1), which encodes the three enzymes in the glyoxylate bypass [21]. The deletion of $i c l R$ in $E$. coli can improve production of phloroglucinol and 3-hydroxypropionate [22], produce succinate from acetate [23], and produce fumaric acid [24, 25], ectoine [26] and fatty acids [27]. The metabolism regulator protein FadR represses the transcription of the $f a d B A$ operon, fadE, fadD, fadH, fadIJ operon, fadL and $f a d M$, which involved in the fatty acid degradation [28]. FadR can also activate the express of gene $i c l R$ and the genes $a c c A, a c c D, a c c B C$ operon, $f a b A, f a b B$ and $f a b H D G$ operon involved in the fatty acid biosynthesis (Fig. 1). Therefore, the absence of FadR can weaken the lipid biosynthesis and enhance the fatty acid degradation in E. coli [29-31]. The iclR deletion mutant strain TWF006 further modified by enhancing fatty acid degradation and the glyoxylate shunt can increase L-threonine production [6]. CpxR and GadE can also activate the genes involved in the dissociated or type II fatty acid synthase systems, such as $f a b A$ and $f a b Z$ [32, 33]. Under the anaerobic conditions, ArcA represses the genes involved in TCA cycle and glyoxylate shunt [34], such as gltA [35], acnAB [36], icd [37], sucABCD [38], lpd [39], sdhCDAB [40], fumA, fumC [41], fumB [42], $m d h$ [43], maeA and $a c e A B$ [44], and the key genes aceEF and $l p d A$ [45] which encoding the enzymes reaction from pyruvate to acetyl-CoA. The L-threonine production in E. coli strain TWF001 was increased when $\operatorname{arc} A$ and $i c l R$ were deleted [46]. Expressing two pyruvate kinase isoenzymes, PYKI and PYKII, encoded by $p y k F$ and pykA, respectively, is important for cellular metabolism and glycolysis in E. coli. In $p y k F$ deletion mutant, the flux of Embden-Meyerhof-Parnas pathway was reduced from 65 to $20 \%$ while the flux of pentose phosphate pathway was increased from 34 to $79 \%$ [47]. Acetate, the main by-product of E. coli at high cell density, can inhibit the cell growth and L-threonine production. Since acetate formation is correlated with the metabolic flux overflow, $p y k F$ deletion can lead to less acetate and more L-threonine production in an L-threonine-producing E. coli THRD [48]. In this study, L-threonine was used to regulate the expression of genes iclR, $\operatorname{arcA}, c p x R, \operatorname{gadE}, f a d R, p y k F$, and $\operatorname{asp} C$ through $t h r L$ and the promoters of $c y s D, c y s H$ and $c y s J$, leading to the increased L-threonine production in $E$. coli TWF001 (Fig. 1). The expression of these seven genes in E. coli are controlled by the concentration of L-threonine. The final strain TWF083 constructed in this study could produce $26.50 \mathrm{~g} / \mathrm{L}$ L-threonine after $21 \mathrm{~h}$ flask fermentation, which was $49.4 \%$ increase compared to the control strain TWF001.

\section{Materials and method Chemicals and reagents}

Plasmids and genomic DNA were extracted using the Plasmids mini kit and DNA purification kit (Tiangen, Beijing, China), respectively. PrimerSTAR HS DNA polymerase (Takara, Dalian, China) was used for PCR. Restriction endonucleases and T4 DNA ligase were purchased from Thermo Scientific (Waltham, USA). Total RNA was extracted using RNA extraction kit (Bio Flux, Beijing, China). The RNA was reversely transcribed into cDNA using the Revert Aid $^{\mathrm{TM}}$ First Strand cDNA synthesis kit (Fermentas, Shanghai, China). RT-PCR was performed using the Real Master Mix kit (Tiangen, Beijing, China). The intracellular concentration of NADPH/ $\mathrm{NADP}^{+}$was determined by using NADPH/NADP ${ }^{+}$Kit (Beyotime Biotechnology, Shanghai, China). 


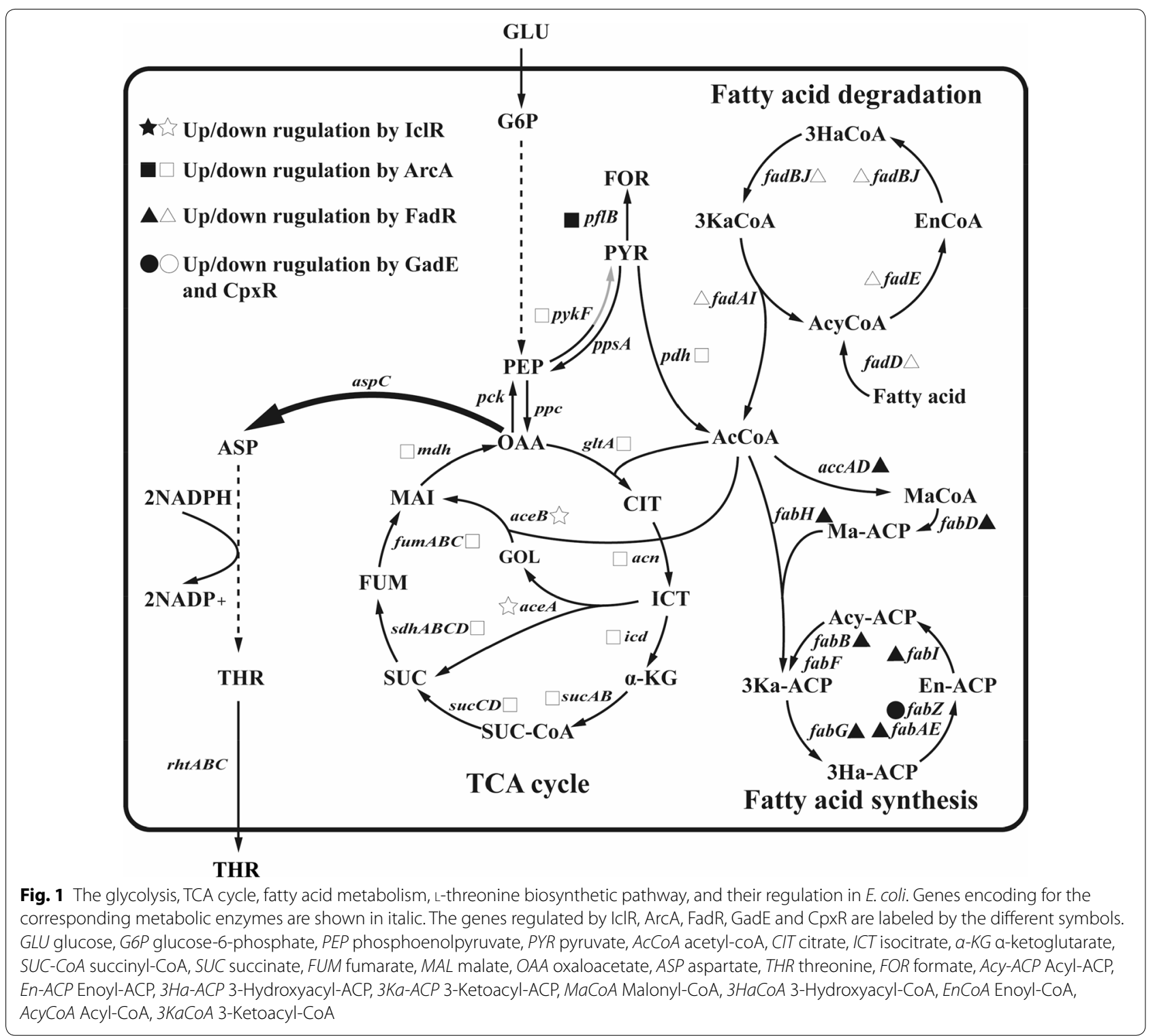

\section{Bacterial growth condition}

The bacterial strains and plasmids used in this study are listed in Table 1. The primers used in this study are listed in Table 2. The promoters used in this study are listed in Table 3. The ribosome binding sites (RBSs) used in this study are listed in Table 4 . The Luria-Bertani (LB) medium (5 g/L yeast extract, $10 \mathrm{~g} / \mathrm{L}$ tryptone, and $10 \mathrm{~g} / \mathrm{L} \mathrm{NaCl}$ ) was used to culture the E. coli cells at $37{ }^{\circ} \mathrm{C}$ or $30^{\circ} \mathrm{C}$ with $200 \mathrm{rpm}$ shaking. To maintain the plasmids in the engineered strains or to provide the selective pressure, kanamycin $(50 \mathrm{mg} / \mathrm{L})$, spectinomycin $(50 \mathrm{mg} / \mathrm{L})$, isopropyl-D-thiogalactopyranoside (IPTG; $0.5 \mathrm{mM})$ or arabinose $(10 \mathrm{mM})$ was supplemented in the medium.
Genome sequence of E. coli MG1655 was used to design the primer sequences because the strain TWF001 [9] was derived from MG1655. The thr operon regulatory region was amplified using the genome sequence of MG1655 as template because the sequence of $t h r$ operon regulatory region in TWF001 was mutated. The insertion fragments were amplified by obtaining the insertion sequence in the first PCR, and then adding the homologous arm in the second PCR. The database on https://salislab.net was used to the design ribosome binding site (RBS) sequence and calculate its translation initiation rate. 
Table 1 Bacterial trains and plasmids used in this study

\begin{tabular}{|c|c|c|}
\hline Strains or plasmids & Description & Sources \\
\hline \multicolumn{3}{|l|}{ Strains } \\
\hline JM109 & Wild type E. coli & NEB \\
\hline MG1655 & Wild type E. coli K-12; $F^{-} \lambda^{-}$rph-1 & CGSC 6300 \\
\hline TWF001 & L-Threonine-producing E. coli strain & [9] \\
\hline TWF003 & TWF001 $\triangle i c I R$ & {$[9]$} \\
\hline TWF010 & TWF001 $\triangle \operatorname{arcA}$ & {$[46]$} \\
\hline TWF051 & $\begin{array}{l}\text { Derived from TWF001 by replacing } R B S_{\text {arcA }} \text { (the } R B S \text { sequence of } \operatorname{arcA} \text { ) with } \operatorname{thr} R\left(R B S_{\text {thr }}-\text { thrL }-R B S_{\text {thra }} \text {, the } R B S\right. \\
\text { sequence of thrL connected with the DNA sequence of the thr operon leader regions, thrL, and followed by the } \\
\text { RBS sequence of thrA) }\end{array}$ & This study \\
\hline TWF052 & Derived from TWF001 by replacing $R B S_{c p \times R}$ with thrR & This study \\
\hline TWF053 & Derived from TWF001 by replacing $R B S_{\text {gadE }}$ with thrR & This study \\
\hline TWF054 & Derived from TWF001 by replacing $R B S_{\text {pyk }}$ with thrR & This study \\
\hline TWF055 & Derived from TWF001 by replacing $R B S_{\text {fadR }}$ with thrR & This study \\
\hline TWF056 & $\begin{array}{l}\text { Derived from TWF001 by inserting the DNA sequence thrR, (the DNA sequence of the thr operon leader regions, } \\
\text { thrL, followed by the } R B S \text { sequence of thrA) between } R B S_{i c l} \text { (the } R B S \text { sequence of } i c / R \text { ) and gene iclR }\end{array}$ & This study \\
\hline TWF057 & $\begin{array}{l}\text { Derived from TWF001 by inserting the DNA sequence thr } R_{2} \text { (the } R B S \text { sequence of thrL, followed by thrL) between } \\
P_{i c / R} \text { (the Promoter sequence of } i c / R \text { ) and } R B S_{i c l R}\end{array}$ & This study \\
\hline TWF058 & Derived from TWF001 by replacing $R B S_{i c l R}$ with the DNA sequence thrR & This study \\
\hline TWF059 & Derived from TWF001 by replacing $P_{i c / R}$ and $R B S_{i c / R}$ with the DNA sequence $P_{\text {thrr }}-$ thrR & This study \\
\hline TWF060 & Derived from TWF058 by replacing $R B S_{i c l R}$ with $\operatorname{thr} R_{s 1}\left(R B S_{t h r L}-t h r L-R B S_{s 1}\right)$ & This study \\
\hline TWF061 & Derived from TWF058 by replacing $R B S_{i c l}$ with $t h r R_{s 2}\left(R B S_{s 2}-t h r L-R B S_{t h r A}\right)$ & This study \\
\hline TWF062 & Derived from TWF058 by replacing $R B S_{i c l}$ with $t h r R_{s 3}\left(R B S_{s 3}-t h r L-R B S_{t h r A}\right)$ & This study \\
\hline TWF063 & Derived from TWF058 by replacing $R B S_{i c l}$ with $t h r R_{s 4}\left(R B S_{s 4}-t h r L-R B S_{t h r A}\right)$ & This study \\
\hline TWF064 & Derived from TWF058 by replacing $R B S_{i c l R}$ with $t h r R_{55}\left(R B S_{55}-t h r L-R B S_{t h r A}\right)$ & This study \\
\hline TWF065 & Derived from TWF058 by replacing $R B S_{i c l R}$ with thrR $R_{s 6}\left(R B S_{s 6}-\right.$ thrL-RBS $\left.S_{\text {thrA }}\right)$ & This study \\
\hline TWF066 & $\begin{array}{l}\text { Derived from TWF063 by inserting the DNA sequence } P_{c y s H}-R B S_{S 7}-a s p C \text { (the Promoter sequence of cys } H \text { followed by a } \\
\text { synthetic } R B S_{s 7} \text { and the gene aspC) in the lacl locus }\end{array}$ & This study \\
\hline TWF067 & Derived from TWF063 by inserting a DNA sequence $P_{c y s}-R B S_{s 8}-a s p C$ in the lacl locus & This study \\
\hline TWF068 & Derived from TWF063 by inserting a DNA sequence $P_{c y s D}-R B S_{s 9}-a s p C$ in the lacl locus & This study \\
\hline TWF069 & Derived from TWF066 by replacing $R B S_{\text {arc }}$ with thrR & This study \\
\hline TWF070 & Derived from TWF066 by replacing $R B S_{c p \times R}$ with thrR & This study \\
\hline TWF071 & Derived from TWF066 by replacing $R B S_{\text {gade }}$ with thrR & This study \\
\hline TWF072 & Derived from TWF066 by replacing $R B S_{\text {pyk }}$ with thrR & This study \\
\hline TWF073 & Derived from TWF066 by replacing RBS $_{\text {fadR }}$ with thrR & This study \\
\hline TWF074 & Derived from TWF070 by replacing $R B S_{\text {arc }}$ with thrR & This study \\
\hline TWF075 & Derived from TWF070 by replacing $R B S_{\text {gadE }}$ with thrR & This study \\
\hline TWF076 & Derived from TWF070 by replacing $R B S_{\text {pyk }}$ with thrR & This study \\
\hline TWF077 & Derived from TWF070 by replacing $R B S_{\text {fadR }}$ with thrR & This study \\
\hline TWF078 & Derived from TWF077 by replacing $R B S_{\text {arc }}$ with thrR & This study \\
\hline TWF079 & Derived from TWF077 by replacing $R B S_{\text {gadE }}$ with thrR & This study \\
\hline TWF080 & Derived from TWF077 by replacing $R B S_{\text {pyk }}$ with thrR & This study \\
\hline TWF081 & Derived from TWF078 by replacing $R B S_{\text {gade }}$ with thrR & This study \\
\hline TWF082 & Derived from TWF078 by replacing $R B S_{\text {pyk }}$ with thrR & This study \\
\hline TWF083 & Derived from TWF081 by replacing $R B S_{\text {pykF }}$ with thrR & This study \\
\hline \multicolumn{3}{|l|}{ Plasmids } \\
\hline pCas9 & repA101(Ts) kan $P_{\operatorname{cas}}$-cas9 $P_{\text {araB }}$-Red lacla $P_{\text {trc }}$-sgRNA-pMB1 & [49] \\
\hline pTargetF & $p M B 1$ aadA sgRNA & [49] \\
\hline pTargetF-arcA & pMB1 aadA sgRNA-arcA & This study \\
\hline pTargetF-cpxR & pMB1 aadA sgRNA- & This study \\
\hline pTargetF-gadE & pMB1 aadA sgRNA-gadE & This study \\
\hline pTargetF-pykF & pMB1 aadA sgRNA-pykF & This study \\
\hline
\end{tabular}


Table 1 (continued)

\begin{tabular}{lll}
\hline Strains or plasmids & Description & Sources \\
\hline pTargetF-fadR & $p M B 1$ aadA sgRNA-fadR & This study \\
pTargetF-iclR1 & $p M B 1$ aadA sgRNA-iclR1 & This study \\
pTargetF-iclR2 & $p M B 1$ aadA sgRNA-iclR2 & This study \\
pTargetF-lacl & $p M B 1$ aadA sgRNA-lacl & This study \\
\hline
\end{tabular}

\section{Construction of E. coli mutant strains TWF051, TWF052, TWF053, TWF054 and TWF055}

The RBS sequence of the five genes $\operatorname{arc} A, c p x R, \operatorname{gad} E$, pykF and $f a d R$ in TWF001 was individually replaced with $\operatorname{thr} R\left(R B S_{t h r L}-t h r L-R B S_{t h r A}\right.$, the combined sequence of the $R B S$ sequence of $t h r L$, thrL, and the $R B S$ sequence of $t h r A$ ). As shown in Fig. 2a, TWF051 was derived from TWF001 by replacing the native RBS of $\operatorname{arcA}$ in chromosome with the thrR-arcA fragments (the thrR fragment with the homologous arm of $\operatorname{arc} A$ ), using the CRISPRCas9 two-plasmid system [49]. Firstly, the fragments of thrR-arcA were amplified using the primer pairs ParcAU-FR1/ParcA-D-RF1and using the genomic DNA of $E$. coli MG1655 as the template, then amplified using the primer pairs ParcA-U-FR11/ParcA-D-RF11and using the recovered fragments as a template. Secondly, the plasmid pTargetF-arc $A$ was constructed though inverse PCR using the primers Pf-ParcA-F/pf-ParcA-R and using pTargetF as the template, and then self-ligation; the primers Pf-ParcA-V-F/pf-V-R was used to confirm the correctness of the plasmid by PCR. Next, pTargetF-arc $A$ and thrR-arcA were used according to the instruction of the CRISPR-Cas9 system [49]. After $36 \mathrm{~h}$ culturing at $30{ }^{\circ} \mathrm{C}$, the correctness of strain was identified using the primer pairs ThrL-V-F/ParcA-V-R, and sequenced using the primers ParcA-S-F/ParcA-S-R. Using the same method, TWF052 was derived from TWF001 by replacing the native RBS of $c p x R$ in chromosome with the $t h r R-c p x R$ fragments. The fragment of thrR-cpxR was amplified using the primer pairs PcpxR-U-FR/PcpxR-D-RF, and then using the primer pairs PcpxR-U-FR11/PcpxR-DRF11. The plasmid pTargetF-cpxR was constructed using the primers Pf-PcpxR-F/pf-PcpxR-R, and the primers $\mathrm{Pf}-\mathrm{P} c p x \mathrm{R}-\mathrm{V}-\mathrm{F} / \mathrm{pf}-\mathrm{V}-\mathrm{R}$ was used to confirm the correctness of the plasmid by PCR. The correctness of strain was identified using the primer pairs ThrL-V-F/PcpxR-V-R, and sequenced using the primers PcpxR-S-F/PcpxR-SR. TWF053 was derived from TWF001 by replacing the native RBS of $c p x R$ in chromosome with the thrR-gadE fragments. The fragments of thrR-gadE were amplified using PgadE-U-FR/PgadE-D-RF, and then using PgadEU-FR11/PgadE-D-RF11. The plasmid pTargetF-gadE was constructed using the primers Pf-PgadE-F/pf-PgadE-R and confirmed using the primers Pf-PgadE-V-F/pf-V-R.
The primer pairs ThrL-V-F/PgadE-V-R and the primers PgadE-S-F/PgadR-S-R were used to identify and sequence the correctness of strain, respectively. TWF054 was derived from TWF001 by replacing the native RBS of $p y k F$ in chromosome with the $t h r R-g a d E$ fragments. The fragments of thrR-pykF were amplified using PpykF-UFR/PpykF-D-RF, and then using PpykF-U-FR11/PpykF$\mathrm{D}-\mathrm{RF} 11$. The plasmid pTargetF-pykF was constructed using the primers Pf-PpykF-F/pf-PpykF-R and confirmed using the primers $\mathrm{Pf}-\mathrm{P} p y k F-\mathrm{V}-\mathrm{F} / \mathrm{pf}-\mathrm{V}-\mathrm{R}$. The primer pairs ThrL-V-F/PpykF-V-R and the primers PpykF-S-F/PpykF$S-R$ were used to identify and sequence the correctness of strain. TWF055 was derived from TWF001 by replacing the native RBS of $f a d R$ in chromosome with the $t h r R-f a d R$ fragments. The fragments of $t h r R-f a d R$ were amplified using PfadR-U-FR/PfadR-D-RF, and then using PfadR-U-FR11/PfadR-D-RF11. The plasmid pTargetF$f a d R$ was constructed using the primers Pf-PfadR-F/pf$\mathrm{P} f a d R-\mathrm{R}$ and confirmed using the primers Pf-PfadR-V-F/ pf-V-R. The primer pairs ThrL-V-F/PfadR-V-R and the primers $\mathrm{P} f a d R-S-\mathrm{F} / \mathrm{P} f a d R-S-R$ were used to identify and sequence the correctness of strain, respectively.

\section{Construction of $E$. coli mutant strains TWF056, TWF057, TWF058, TWF059, TWF060, TWF061, TWF062, TWF063, TWF064 and TWF065}

As shown in Fig. 3a, TWF056 was derived from TWF001 by inserting the $t h r L-R B S_{t h r A}\left(t h r R_{1}\right)$ fragment between the native ribosomal binding site of the $i c l R\left(R B S_{i c l R}\right)$ and the $i c l R$ sequence, using the CRISPR-Cas9 two-plasmid system [49]. Firstly, the $t h r R_{1}$ fragment was amplified using the primer pairs PiclR-U-FR1/PiclR-D-RF1 and using the genomic DNA of MG1655 as a template, and then amplified using the primer pairs PiclR-U-FR11/ PiclR-D-RF1 and using the recovered fragments as a template. Secondly, the plasmid pTargetF-iclR1 was constructed using the primers Pf-PiclR-F/pf-PiclR-R by inverse PCR using pTargetF as template, and then selfligation. The primers Pf-PiclR-V-F/pf-V-R was using to confirm the correctness of the plasmid by PCR. The primer pairs ThrL-V-F/PiclR-V-R and the primers PiclR-S-F/PiclR-S-R were used to identify and sequence the correctness of strain, respectively. Using the same method, TWF057 was constructed. $R B S_{t h r L}$-thrL $\left(t h r R_{2}\right)$ 
Table 2 The primers used in this study

\begin{tabular}{|c|c|c|}
\hline Names & Sequence $\left(5^{\prime}-3^{\prime}\right)$ & Purpose \\
\hline ParcA-U-FR1 & $\begin{array}{l}\text { TGTTAATTTGCAGCATGCATCAGGCAGGTCAGGGACTTTTGATAG } \\
\text { CGCACAGACAGATAAAAATTACA }\end{array}$ & \multirow[t]{4}{*}{ The thrR-arcA fragments with the homologous arm of $\operatorname{arc} A$} \\
\hline ParcA-D-RF1 & $\begin{array}{l}\text { TGTTACCAACTCGTCTTCAACGATAAGAATGTGCGGGGTCTGCAT } \\
\text { GGTTGTTACCTCGTTACCTTTGG }\end{array}$ & \\
\hline ParcA-U-FR11 & $\begin{array}{l}\text { AACGCAATTACGTACTTTAGTCATGTTACGCCGATCATGTTAATTTGC } \\
\text { AGCATGCATCA }\end{array}$ & \\
\hline ParcA-D-RF11 & $\begin{array}{l}\text { GCCTTCCGCTTCGAAAATACTTTTCAACGTGTTGCGTGTTACCAA } \\
\text { CTCGTCTTCAACGA }\end{array}$ & \\
\hline Pf-ParcA-F & $\begin{array}{l}\text { GTCCTAGGTATAATACTAGT TTCGATTTAGTTGGCAATTTGTTTTAG } \\
\text { AGCTAGAAATAG }\end{array}$ & \multirow[t]{3}{*}{ pTargetF-arcA } \\
\hline pf-ParcA-R & $\begin{array}{l}\text { CTATTTCTAGCTCTAAAACAAATTGCCAACTAAATCGAAACTAGTATT } \\
\text { ATACCTAGGAC }\end{array}$ & \\
\hline Pf-ParcA-V-F & TTCGATTTAGTTGGCAATTT & \\
\hline ParcA-V-R & CGGGTTGAACGGTTTGGTG & \multirow[t]{3}{*}{ Identify and sequence TWF051, TWF069, TWF074 and TWF078 } \\
\hline ParcA-S-F & CGGGTTGAACGGTTTGGTG & \\
\hline ParcA-S-R & CGGGTTGAACGGTTTGGTG & \\
\hline PcpxR-U-FR & $\begin{array}{l}\text { AAGTCATGGATTAGCGACGTCTGATGACGTAATTTCTGCCTATAGCGC } \\
\text { ACAGACAGATAAAAATTACA }\end{array}$ & \multirow[t]{4}{*}{ The thrR-cpxR fragments with the homologous arm of $c p x R$} \\
\hline PcpxR-D-RF & $\begin{array}{l}\text { GGAAGTCAGCTCTCGGTCATCATCAACTAACAGGATTTTATTCATGGT } \\
\text { TGTTACCTCGTTACCTTTGG }\end{array}$ & \\
\hline PcpxR-U-FR11 & $\begin{array}{l}\text { GCTGCAAACATGCGTCAGGGGGTGTAAAACAACGTAAAGTCATGG } \\
\text { ATTAGCGACGTCTG }\end{array}$ & \\
\hline PcpxR-D-RF11 & $\begin{array}{l}\text { CGTTGAAGCCTTCCATCTCGAGCAGCTCCTTTAATAGGGAAGTCA } \\
\text { GCTCTCGGTCATCA }\end{array}$ & \\
\hline Pf-PcpxR-F & $\begin{array}{l}\text { GTCCTAGGTATAATACTAGTTGACGTAATTTCTGCCTCGGGTTTTAGA } \\
\text { GCTAGAAATAG }\end{array}$ & \multirow[t]{3}{*}{ pTargetF-cpxR } \\
\hline pf-PcpxR-R & $\begin{array}{l}\text { CTATTTCTAGCTCTAAAACCCGAGGCAGAAATTACGTCAACTAGTATT } \\
\text { ATACCTAGGAC }\end{array}$ & \\
\hline Pf-PcpxR-V-F & TGACGTAATTTCTGCCTCGG & \\
\hline PcpxR-V-R & GCCGCCACCACATTAA & \multirow[t]{3}{*}{ Identify and sequence TWF052 and TWF070 } \\
\hline PcpxR-S-F & AGCAGCGTGGCTTAATGAACT & \\
\hline PcpxR-S-R & CATAATGACAGGCGTCTGGTGT & \\
\hline PgadE-U-FR & $\begin{array}{l}\text { ACAAGGATGTAAATAATGAAAAGGATGACATATTCGAAACGATAG } \\
\text { CGCACAGACAGATAAAAATTACA }\end{array}$ & \multirow[t]{4}{*}{ The thrR-gadE fragments with the homologous arm of gadE } \\
\hline PgadE-D-RF & $\begin{array}{l}\text { AAAGCCCTGTAAAAGAAAAGAATCTTTCGTCATGAGAAAAATCAT } \\
\text { GGTTGTTACCTCGTTACCTTTGG }\end{array}$ & \\
\hline PgadE-U-FR11 & $\begin{array}{l}\text { ATAGGCGTTTACTATATTGAACAACGATTCGGACAAGGATGTAAATAA } \\
\text { TGAAAAGGATG }\end{array}$ & \\
\hline PgadE-D-RF11 & $\begin{array}{l}\text { TTTTATCATTTCGTGATTATCTTTCAACTGCCAAAAGCCCTGTAAAAG } \\
\text { AAAAGAATCTT }\end{array}$ & \\
\hline Pf-PgadE-F & $\begin{array}{l}\text { GTCCTAGGTATAATACTAGTATTCGAAACGATAACGGCTAGTTTTAGA } \\
\text { GCTAGAAATAG }\end{array}$ & \multirow[t]{3}{*}{ pTargetF-gadE } \\
\hline pf-PgadE-R & $\begin{array}{l}\text { CTATTTCTAGCTCTAAAACTAGCCGTTATCGTTTCGAATACTAGTATT } \\
\text { ATACCTAGGAC }\end{array}$ & \\
\hline Pf-PgadE-V-F & ATTCGAAACGATAACGGCTA & \\
\hline PgadE-V-R & GTTCCTGCCAGCATTCG & \multirow{3}{*}{$\begin{array}{l}\text { Identify and sequence TWF053, TWF071, TWF075, TWF079 and } \\
\text { TWF081 }\end{array}$} \\
\hline PgadE-S-F & TGATAACTTATTCTTGGGCAGTA & \\
\hline PgadE-S-R & TTCATCAAGGATATGATTGTG & \\
\hline
\end{tabular}


Table 2 (continued)

\begin{tabular}{|c|c|c|}
\hline Names & Sequence $\left(5^{\prime}-3^{\prime}\right)$ & Purpose \\
\hline PpykF-U-FR & $\begin{array}{l}\text { ACCGGATTCGCTTTCCGGCAGTGCGCCCAGAAAGCAAGTTTATAG } \\
\text { CGCACAGACAGATAAAAATTACA }\end{array}$ & \multirow[t]{4}{*}{ The thrR-pykF fragments with the homologous arm of pykF } \\
\hline PpykF-D-RF & $\begin{array}{l}\text { TTCGGTTTTCGGTCCGATGGTGCAAACAATTTTGGTCTTTTTTCATGGT } \\
\text { TGTTACCTCGTTACCTTTGG }\end{array}$ & \\
\hline PpykF-U-FR11 & $\begin{array}{l}\text { TGTCACCTATCCTTAGAGCGAGGCACCACCACTTTCGTAATACCG } \\
\text { GATTCGCTTTCCGG }\end{array}$ & \\
\hline PpykF-D-RF11 & $\begin{array}{l}\text { TCATGCCAGCGTCCAGCATTTTAGCTAACATCTCTTCAGATTCGGTTT } \\
\text { TCGGTCCGATG }\end{array}$ & \\
\hline Pf-PpykF-F & $\begin{array}{l}\text { GTCCTAGGTATAATACTAGTTCTTAGTCTTTAAGTTGAGAGTTTTAGA } \\
\text { GCTAGAAATAG }\end{array}$ & \multirow[t]{3}{*}{ pTargetF-pykF } \\
\hline pf-PpykF-R & $\begin{array}{l}\text { CTATTTCTAGCTCTAAAACTCTCAACTTAAAGACTAAGAACTAGTATT } \\
\text { ATACCTAGGAC }\end{array}$ & \\
\hline Pf-PpykF-V-F & TCTTAGTCTTTAAGTTGAGA & \\
\hline PpykF-V-R & ACTTCCATACCGATCAGACCAT & \multirow{3}{*}{$\begin{array}{l}\text { Identify and sequence TWF054, TWF072, TWF076, TWF080, TWF082 } \\
\text { and TWF083 }\end{array}$} \\
\hline PpykF-S-F & TGACAACTTCGGCACCAGA & \\
\hline PpykF-S-R & GTCGTTACCGCCTTCCAGT & \\
\hline PfadR-U-FR & $\begin{array}{l}\text { GCGTAGTTAGCCCTCTGGTATGATGAGTCCAACTTTGTTTATAGCGCA } \\
\text { CAGACAGATAAAAATTACA }\end{array}$ & \multirow[t]{4}{*}{ The thrR-fadR fragments with the homologous arm of fadR } \\
\hline PfadR-D-RF & $\begin{array}{l}\text { GAAACCCGCCGGGCTTTGCGCCTTAATGACCATGGTTGTTACCTC } \\
\text { GTTACCTTTGG }\end{array}$ & \\
\hline PfadR-U-FR1 1 & $\begin{array}{l}\text { GCCTTGATCCCTTTTTCTTCTTTTTGTTCTGCTATCAGCGTAGTTAGCC } \\
\text { CTCTGGTATGA }\end{array}$ & \\
\hline PfadR-D-RF11 & $\begin{array}{l}\text { AAGCGGTTATTCCAGATACTTTCAATAATGTACTCTTCCGCGAAACCC } \\
\text { GCCGGGCTTTG }\end{array}$ & \\
\hline Pf-PfadR-F & $\begin{array}{l}\text { GTCCTAGGTATAATACTAGTAACTTTGTTTTGGCTGTGTTAGTTTTAGA } \\
\text { GCTAGAAATAG }\end{array}$ & \multirow[t]{3}{*}{ pTargetF-fadR } \\
\hline pf-PfadR-R & $\begin{array}{l}\text { CTATTTCTAGCTCTAAAACTAACACAGCAAAACAAAGTTACTAGTATT } \\
\text { ATACCTAGGAC }\end{array}$ & \\
\hline Pf-PfadR-V-F & AACTTTGTTTTGCTGTGTTA & \\
\hline PfadR-V-R & GCCAAATCTCGCCACTC & \multirow[t]{3}{*}{ Identify and sequence TWF055, TWF073 and TWF077 } \\
\hline PfadR-S-F & GAGATCTCCATGATGGTTTCCCTTA & \\
\hline PfadR-S-R & GAGATCTCCATGATGGTTTCCCTTA & \\
\hline PiclR-U-FR1 & $\begin{array}{l}\text { TGAAAATGATTTCCACGATACAGAAAAAAGAGACTGTCATGAAAC } \\
\text { GCATTAGCACCACC }\end{array}$ & \multirow[t]{4}{*}{ The $t h r R_{1}$ fragments with the homologous arm of iclR } \\
\hline PiclR-D-RF1 & $\begin{array}{l}\text { TTTTCTGCCGCGTTTCGCGGGAATGGGTGCGACCATGGTTGTTAC } \\
\text { CTCGTTACCTTTGG }\end{array}$ & \\
\hline PiclR-U-FR11 & $\begin{array}{l}\text { GTTCAGTAACTATTGCATTAGCTAACAATAAAAATGAAAATGATTTCC } \\
\text { ACGATACAGAA }\end{array}$ & \\
\hline PiclR-D-RF11 & $\begin{array}{l}\text { GACTGAACCTGTCCAGTCGCTGGTGCGGTGGCAACGGCGGGTTTT } \\
\text { CTGCCGCGTTTCGC }\end{array}$ & \\
\hline Pf-PiclR-F & $\begin{array}{l}\text { GTCCTAGGTATAATACTAGTACAGAAAAAAGAGACTGTCAGTTTT } \\
\text { AGAGCTAGAAATAG }\end{array}$ & \multirow[t]{4}{*}{ pTargetF-iclR1 } \\
\hline pf-PiclR-R & $\begin{array}{l}\text { CTATTTCTAGCTCTAAAACTGACAGTCTCTTTTTTCTGTACTAGTATT } \\
\text { ATACCTAGGAC }\end{array}$ & \\
\hline Pf-PiclR-V-F & ACAGAAAAAAGAGACTGTCA & \\
\hline pf-V-R & TTGTCAGCAAGATAGCCAGA & \\
\hline ThrL-V-F & ATGAAACGCATTAGCACC & \multirow{4}{*}{$\begin{array}{l}\text { Identify and sequence TWF056, TWF057, TWF058, TWF059, TWF060, } \\
\text { TWF061, TWF062, TWF063, TWF064 and TWF065 }\end{array}$} \\
\hline PiclR-V-R & CACCTGTTCTTCGCTCAGTTGG & \\
\hline PiclR-S-F & GTAGGCGTTGTGGATAGCGG & \\
\hline PiclR-S-R & TTGCCCAATGTCCCAGTTCG & \\
\hline
\end{tabular}


Table 2 (continued)

\begin{tabular}{|c|c|c|}
\hline Names & Sequence $\left(5^{\prime}-3^{\prime}\right)$ & Purpose \\
\hline PicIR-U-FR2 & $\begin{array}{l}\text { ATTAGCTAACAATAAAAATGAAAATGATTTCCATAGCGCACAGAC } \\
\text { AGATAAAAATTACA }\end{array}$ & The thr $R_{2}$ fragments with the homologous arm of iclR \\
\hline PiclR-D-RF2 & $\begin{array}{l}\text { ATGGGTGCGACCATGACAGTCTCTTTTTTCTGTATCGTAAAAAAA } \\
\text { AAGCCCGCACTGTC }\end{array}$ & \\
\hline PiclR-U-FR22 & $\begin{array}{l}\text { ACTCATCGGATCAGTTCAGTAACTATTGCATTAGCTAACAATAAAAAT } \\
\text { GAAAATGATTT }\end{array}$ & \\
\hline PiclR-D-RF22 & $\begin{array}{l}\text { GCGGTGGCAACGGCGGGTTTTCTGCCGCGTTTCGCGGGAATGGGT } \\
\text { GCGACCATGACAGT }\end{array}$ & \\
\hline Pf-PiclR-F2 & $\begin{array}{l}\text { GTCCTAGGTATAATACTAGTAGTCTCTTTTTTCTGTATCGGTTTTAGA } \\
\text { GCTAGAAATAG }\end{array}$ & pTargetF-icIR2 \\
\hline pf-PicIR-R2 & $\begin{array}{l}\text { CTATTTCTAGCTCTAAAACCGATACAGAAAAAAGAGACTACTAGTATT } \\
\text { ATACCTAGGAC }\end{array}$ & \\
\hline Pf-PiclR-V-F2 & AGTCTCTTTTTTCTGTATCG & \\
\hline PiclR-U-FR3 & $\begin{array}{l}\text { CACCACGCAACATGAGATTTGTTCAACATTAAAAAACGCCTTAGT } \\
\text { AAGTATTTTTCAGC }\end{array}$ & $P_{\text {thri }}$ - thrR fragments with the homologous arm of iclR \\
\hline PiclR-U-FR33 & $\begin{array}{l}\text { ACCATACTGGCATAAACGCATCTGTGGTAAAAGCGACCACCACGC } \\
\text { AACATGAGATTTGT }\end{array}$ & \\
\hline PiclR-D-RF4 & $\begin{array}{l}\text { TTTCGCGGGAATGGGTGCGACCATTGAGATACCTCTTCTTATTTTTCT } \\
\text { GAGCAAAAAAAAAGCCCGCACTGTC }\end{array}$ & The $t h r R_{s 1}$ fragments with the homologous arm of iclR \\
\hline PicIR-D-RF44 & $\begin{array}{l}\text { CCTGTCCAGTCGCTGGTGCGGTGGCAACGGCGGGTTTTCTGCCGC } \\
\text { GTTTCGCGGGAATGGGTGC }\end{array}$ & \\
\hline PiclR-U-FR5 & $\begin{array}{l}\text { ATTAGCTAACAATAAAAATGAAAATGATTTCCACCAAAACCATAA } \\
\text { GGAGGATTTACATGAAACGCATTAGCACCACC }\end{array}$ & $\begin{array}{l}\text { The } t h r R_{s 2} \\
\text { fragments with the homologous arm of iclR }\end{array}$ \\
\hline PiclR-U-FR55 & $\begin{array}{l}\text { ACATGAGATTTGTTCAACATTAACTCATCGGATCAGTTCAGTAACTAT } \\
\text { TGCATTAGCTAACAATAAAAATGAAAATGATTT }\end{array}$ & \\
\hline PiclR-U-FR6 & $\begin{array}{l}\text { ATTAGCTAACAATAAAAATGAAAATGATTTCCGTAGCTGAGACTCTAA } \\
\text { GGGAGGCGTCACATGAAACGCATTAGCACCACC }\end{array}$ & The $t h r R_{s 3}$ fragments with the homologous arm of iclR \\
\hline PiclR-U-FR7 & $\begin{array}{l}\text { ATTAGCTAACAATAAAAATGAAAATGATTTCCGCATACATTCCACGAC } \\
\text { CATAGGAACCAACCCATGAAACGCATTAGCACCACC }\end{array}$ & The $t h r R_{s 4}$ fragments with the homologous arm of iclR \\
\hline PiclR-U-FR8 & $\begin{array}{l}\text { ATTAGCTAACAATAAAAATGAAAATGATTTCCAAGCCCATTTTACCAT } \\
\text { CGAAGAGGGACAGAATGAAACGCATTAGCACCACC }\end{array}$ & The $t h r R_{s 5}$ fragments with the homologous arm of iclR \\
\hline PiclR-U-FR9 & $\begin{array}{l}\text { ATTAGCTAACAATAAAAATGAAAATGATTTCCTCCTTTTAGGGCTATC } \\
\text { GCGGAGGAGTAATGAAACGCATTAGCACCACC }\end{array}$ & The $t h r R_{s 6}$ fragments with the homologous arm of iclR \\
\hline cysH-aspC-F2 & TAGCCACCTAGGAGGACTCCATGTTTGAGAACATTACCGCCG & The $R B S_{S 7}$-aspC fragment \\
\hline cysH-aspC-R2 & $\begin{array}{l}\text { CTTCCACTTTTTTCCCGCGTTITCGCAGAAACGTGGCTGGCCTGGT } \\
\text { TCACCACGCGGGAAACGGTCTGATAGGCACATAAAAAAGCCCGC }\end{array}$ & \\
\hline cysH-aspC-F1 & $\begin{array}{c}\text { ACCTTTCGCGGTATGGCATGATAGCGCCCGGAAGAGAGTCAATTC } \\
\text { AGGGTGGTGAATGTGAAACCAGTACGGAAATCCTGGCGTCGC }\end{array}$ & The promoter of $c y s H$ \\
\hline cysH-aspC-R1 & GGAGTCCTCCTAGGTGGCTATGCCTTGCCTGATGCGAC & \\
\hline cys-aspC-F & $\begin{array}{l}\text { TTCTGGTGGCCGGAAGGCGAAGCGGCATGCATTTACGTTGACACC } \\
\text { ATCGAATGGCGCAAAACCTTTCGCGGTATGGCAT }\end{array}$ & The $P_{\text {cysH }}-R B S_{s 7}$-aspC fragment with the homologous arm of lacl \\
\hline cys-aspC-R & $\begin{array}{l}\text { TGTTTGCCCGCCAGTTGTTGTGCCACGCGGTTGGGAATGTAATTC } \\
\text { AGCTCCGCCATCGCCGCTTCCACTTTTTCCCGCG }\end{array}$ & \\
\hline Pf-lacl-F & $\begin{array}{l}\text { GTCCTAGGTATAATACTAGTTACGATGTCGCAGAGTATGCGTTTTAGA } \\
\text { GCTAGAAATAG }\end{array}$ & pTargetF-lacl \\
\hline pf-lacl-R & $\begin{array}{l}\text { CTATTTCTAGCTCTAAAACGCATACTCTGCGACATCGTAACTAGTATT } \\
\text { ATACCTAGGAC }\end{array}$ & \\
\hline Pf-lacl-V-F & TACGATGTCGCAGAGTATGC & \\
\hline lacl-aspC-V-F & CCTACGCTGGAACAATGGCAAACACTGGCACA & Identify and sequence TWF066, TWF067 and TWF068 \\
\hline lacl-aspC-V-R & GCTTCCACAGCAATGGCATCCTGGTCATCC & \\
\hline lacl-S-F & AACAGATCGAAGAAGGGGTTGA & \\
\hline lacl-S-R & GACGGCGCGTGCAGGGCC & \\
\hline
\end{tabular}


Table 2 (continued)

\begin{tabular}{|c|c|c|}
\hline Names & Sequence $\left(5^{\prime}-3^{\prime}\right)$ & Purpose \\
\hline cysJ-aspC-F2 & $\begin{array}{l}\text { CGCATAACAATTCGTATCATAAGGAGACCATATGTTTGAGAACATTAC } \\
\text { CGCCG }\end{array}$ & The $P_{c y s} J^{-R B S_{s 8}}$-aspC fragment with the homologous arm of lacl \\
\hline cysJ-aspC-F1 & $\begin{array}{l}\text { ACCTTTCGCGGTATGGCATGATAGCGCCCGGAAGAGAGTCAATTC } \\
\text { AGGGTGGTGAATGTGAAACCAGTAGTTGCGCAAAATCGCTGATT }\end{array}$ & \\
\hline cysJ-aspC-R1 & $\begin{array}{l}\text { ATGGTCTCCTTATGATACGAATTGTTATGCGGTAAGCAAAGCTGTTTC } \\
\text { TGCGC }\end{array}$ & \\
\hline cysD-aspC-F2 & $\begin{array}{l}\text { AAGGCCAAAGACACTAAGAATTATTTATATGTTTGAGAACATTAC } \\
\text { CGCCG }\end{array}$ & The $P_{c y s D}-R B S_{s 9}-a s p C$ fragment with the homologous arm of lacl \\
\hline cysD-aspC-F1 & $\begin{array}{l}\text { CCTTTCGCGGTATGGCATGATAGCGCCCGGAAGAGAGTCAATTCA } \\
\text { GGGTGGTGAATGTGAAACCAGTACGGTGCCTTAAGCACTTTTTG }\end{array}$ & \\
\hline cysD-aspC-R1 & $\begin{array}{l}\text { ATAAATAATTCTTAGTGTCTTTGGCCTTATGTTTCGACTATAGGGAGC } \\
\text { GTAAG }\end{array}$ & \\
\hline RT-16SrDNA-F & TTTAATTCGATGCAACGCGAAGAACC & Transcriptional level \\
\hline RT-16SrDNA-R & CGGACCGCTGGCAACAAAGGATAAG & \\
\hline RT-icIR-F & CAGGGTTTCGTGCGTCAGGTTGG & \\
\hline RT-iclR-R & CGATAATAATCGCTTCGTGATCGCTTTG & \\
\hline RT-aspC-F & GAGAACATTACCGCCGCTCCTGC & \\
\hline $\mathrm{RT}-\operatorname{aspC-R}$ & CCCGTCTCATCTTTATAGACACCAATCCC & \\
\hline RT-fadR-F & GGAAGTGCTGGCTACCGCTAATGAAG & \\
\hline RT-fadR-R & CATCCCGTTAAGAATCAGACCGTAAATCG & \\
\hline $\mathrm{RT}-c p \times R-\mathrm{F}$ & ACAGCATCTGGGTCAGGTGGTTTCC & \\
\hline $\mathrm{RT}-c p \times R-\mathrm{R}$ & CGGCAGTTTACGACGCAGGTTGG & \\
\hline RT-gadE-F & AGGCAATAAACCCTTCAAG & \\
\hline RT-gadE-R & TCGGCATCTAATTTCTCCAG & \\
\hline RT-pykF-F & GCGTTTCCATTGCTCTGCC & \\
\hline RT-pykF-R & GCTTTCAGGTGCTCACGGATTTC & \\
\hline RT-arcA-F & AGTCCCGTGCTGAACTGCTGAAG & \\
\hline RT-arcA-R & ACCGTGAATGGTGGCGATG & \\
\hline
\end{tabular}

Table 3 The promoters used in this study

\begin{tabular}{|c|c|}
\hline Promoter & Sequence $\left(5^{\prime}-3^{\prime}\right)$ \\
\hline thrL & GCCGTGAGTAAATTAAAATTTTATTGACTTAGGTCACTAAATACTTTAACCAATATAGGC \\
\hline$i c / R$ & CTCATCGGATCAGTTCAGTAACTATTGCATTAGCTAACAATAAAAATGAAAATGATTTCC \\
\hline CysH & $\begin{array}{l}\text { CGGAAATCCTGGCGTCGCTTGATGAACTGATAGGGCGCTGGGCGAAAGAGCGCGAAGCGGGTGAAGGCTTCGGCGACTTTACGGTGCGTGCGGGCA } \\
\text { TCATTCGCCCGGTGCTCGATCCGGCGCGTGATTTGTGGGATTAACCATCAGCCCGGTCTTGTAGGCCTGATAAGAACGCGTGAGCGTCGCATCAGG } \\
\text { CAAGGCA }\end{array}$ \\
\hline cys」 & $\begin{array}{l}\text { GTTGCGCAAAATCGCTGATTTATCTTAATGATTGGCTAAATTCATTTGTTTTTTCATTAGGTTGGTTAATCTATTTTGGTTGTTAAAGACTATTGCTAAAACAG } \\
\text { GTTAGTCGATTTGGTTATTAGTTATCGCTATCCCGTCTTTAATCCACACCGTTTGCCCCGTTAACCTTACCTTCTCTTCTGTTTAATGGCGCTGACAG } \\
\text { GGCGCAGAAACAGCTTTGCTTAC }\end{array}$ \\
\hline cysD & $\begin{array}{l}\text { CGGTGCCTTAAGCACTTTTTGATATTAGCTTTGCCAAATCGTTATTCCGTTAAGGAACTACTCATTCTAATTGGTAATTTCATTCGTTCTCTTACGCTCCCT } \\
\text { ATAGTCGAAACAT }\end{array}$ \\
\hline $\operatorname{arcA}$ & ATCATGTTAATTTGCAGCATGCATCAGGCAGGTCAGGGACTTTTG \\
\hline$c p \times R$ & GGGGGTGTAAAACAACGTAAAGTCATGGATTAGCGACGTCTGATGACGTAATTTCTGCCT \\
\hline gadE & ATATTGAACAACGATTCGGACAAGGATGTAAATAATGAAAAGGATGACATATTCGAAACG \\
\hline pykF & $\begin{array}{l}\text { ATATTTTTTGAAACGCTGTTTTTGTTTTCCTTTTGGATTAATTTCAGCGTATAATGCGCGCCAATTGACTCTTGAATGGTTTCAGCACTTTGGACTGTAGAA } \\
\text { CTCAACGACTCAAAAACAGGCACTCACGTTGGGCTGAGACACAAGCACACATTCCTCTGCACGCTTTTTCGATGTCACCTATCCTTAGAGCGAGGC } \\
\text { ACCACCACTTTCGTAATACCGGATTCGCTTTCCGGCAGTGCGCCCAGAAAGCAAGTTT }\end{array}$ \\
\hline fadR & ATCCCTTTTTCTTCTTTTTGTCTGCTATCAGCGTAGTTAGCCCTCTGGTATGATGAGTCCAACTTTGTTT \\
\hline
\end{tabular}


Table 4 The ribosome binding sites used in this study

\begin{tabular}{|c|c|c|}
\hline Names & Sequence $\left(5^{\prime}-3^{\prime}\right)$ & $\begin{array}{l}\text { RBS Calculator's } \\
\text { proportional scale } \\
\text { (au) [64] }\end{array}$ \\
\hline$R_{B S} S_{\text {thrl }}$ & ATAGCGCACAGACAGATAAAAATTACAGAGTACACAACATCC & - \\
\hline$R B S_{\text {thrA }}$ & CGACCAAAGGTAACGAGGTAACAACC & - \\
\hline$R B S_{i c l R}$ & ACGATACAGAAAAAAGAGACTGTC & - \\
\hline$R_{B S}$ & GCTCAGAAAAATAAGAAGAGGTATCTCA & 3800 \\
\hline$R B S_{S 2}$ & ACCAAAACCATAAGGAGGATTTAC & 380,000 \\
\hline$R_{B S} S_{53}$ & GTAGCTGAGACTCTAAGGGAGGCGTCAC & 45000 \\
\hline$R_{B S} S_{4}$ & GCATACATTCCACGACCATAGGAACCAACCC & 15,000 \\
\hline$R B S_{55}$ & AAGCCCATTTTACCATCGAAGAGGGACAGA & 5000 \\
\hline$R B S_{s 6}$ & TCCTTTTAGGGCTATCGCGGAGGAGTA & 1000 \\
\hline$R B S_{S 7}$ & TAGCCACCTAGGAGGACTCC & 2000 \\
\hline$R_{B S} S_{8}$ & CGCATAACAATTCGTATCATAAGGAGACCAT & 2000 \\
\hline$R_{B S} S_{s 9}$ & AAGGCCAAAGACACTAAGAATTATTTAT & 2000 \\
\hline$R B S_{\text {arcA }}$ & TACTTCCTGTTTCGATTTAGTTGGCAATTTAGGTAGCAAAC & - \\
\hline$R B S_{c p \times R}$ & CGGAGGTATTTAAACA & - \\
\hline$R B S_{\text {gadE }}$ & ATAACGGCTAAGGAGCAAGTT & - \\
\hline$R B S_{p y k F}$ & СTCCCATCCTTCTCAACTTAAAGACTAAGACTGTC & - \\
\hline$R B S_{\text {fadR }}$ & TGCTGTGTTATGGAAATCTCACT & - \\
\hline
\end{tabular}

was amplified using the primers PiclR-U-FR2/PiclRD-RF2, and then amplified using the PiclR-U-FR22/ PiclR-D-RF22. The plasmid pTargetF-iclR2 was constructed using the primers Pf-PiclR-F2/pf-PiclR-R2, and identified using the primers Pf-PiclR-V-F2/pf-V-R). To construct TWF058, the sequence $R B S_{t h r}-t h r L-R B S_{t h r A}$ (thrR) was amplified using PiclR-U-FR2/PiclR-D-RF1, and then amplified using PiclR-U-FR22/PiclR-D-RF11. The plasmid pTargetF-iclR1 was used. To constructed TWF059, the sequence $P_{t h r L}$-thrR was amplified using PiclR-U-FR3/PiclR-D-RF1, and then amplified using PiclR-U-FR33/PiclR-D-RF11. The plasmid pTargetF-iclR1 was used. To construct TWF060, the sequence $R B S_{t h r L^{-}}$ thrL-RBS $S_{s 1}\left(t h r R_{s 1}\right)$ was amplified using PiclR-U-FR2/ PiclR-D-RF4, and then amplified using PiclR-U-FR22/ PiclR-D-RF44. The plasmid pTargetF-iclR1 was used. To construct TWF061, the sequence $R B S_{s 2}-t h r L-R B S_{t h r}$ $\left(t h r R_{s 2}\right)$ was amplified using PiclR-U-FR5/PiclR-D-RF4, and then amplified using PiclR-U-FR55/PiclR-D-RF44. The plasmid pTargetF-iclR1 was used. To construct TWF062, the sequence $R B S_{s 3}-t h r L-R B S_{t h r A}\left(t h r R_{s 3}\right)$ was amplified using PiclR-U-FR6/PiclR-D-RF4, and then amplified using PiclR-U-FR55/PiclR-D-RF44. The plasmid pTargetF-iclR1 was used. To construct TWF063, the sequence $R B S_{s 4}$-thrL-RBS $S_{t h r}\left(t h r R_{s 4}\right)$ was amplified using PiclR-U-FR7/PiclR-D-RF4, and then amplified using PiclR-U-FR55/PiclR-D-RF44. The plasmid pTargetF-iclR1 was used. To construct TWF064, the sequence $R B S_{55^{-}}$ thrL-RBS $S_{t h r A}\left(t h r R_{s 5}\right)$ was amplified using PiclR-U-FR8/
PiclR-D-RF4, and then amplified using PiclR-U-FR55/ PiclR-D-RF44. The plasmid pTargetF-iclR1 was used. To construct TWF065, the sequence $R B S_{s 6}-t h r L-R B S_{t h r A}$ $\left(t h r R_{s 6}\right)$ was amplified using PiclR-U-FR9/PiclR-D-RF4, and then amplified using PiclR-U-FR55/PiclR-D-RF44. The plasmid pTargetF-iclR1 was used.

Construction of $E$. coli mutant strains TWF066, TWF067, TWF068, TWF069, TWF070, TWF071, TWF072, TWF073, TWF074, TWF075, TWF076, TWF077, TWF078, TWF079, TWF080, TWF081, TWF082 and TWF083

As shown in Fig. 4a, TWF066 was derived from TWF063 by inserting the gene $\operatorname{asp} C$ controlled by the promoter of cysH $\left(P_{c y s H}\right)$ and $R B S_{s 7}$ into the lacI locus in chromosome. The fragment of $R B S_{s}-a s p C$ was amplified by using the primers cysH-aspC-F2/cysH-aspC-R2 and using the genomic DNA of TWF001 as a template. The promoter of $c y s H$ was amplified by using the primers cysH-aspC-F1/cysH-aspC-R1 and using the genomic DNA of TWF001 as template. These two DNA fragments were linked together by overlap PCR, using the primers cys-aspC-F/cys-aspC-R, and resulting in the replacement fragment $P_{c y s H^{-}} R B S_{s 7}-a s p C$. The plasmid pTargetF-lacI was constructed using Pf-lacI-F/pf-lacI-R, and confirmed using Pf-lacI-V-F/pf-V-R. The primers lacIaspC-V-F/lacI-aspC-V-R and lacI-S-F/lacI-S-R were used to identify and sequence the correctness of the strain, respectively. TWF067 was constructed from TWF001 by inserting the gene $\operatorname{aspC}$ controlled by the promoter of $c y s J$ 
a

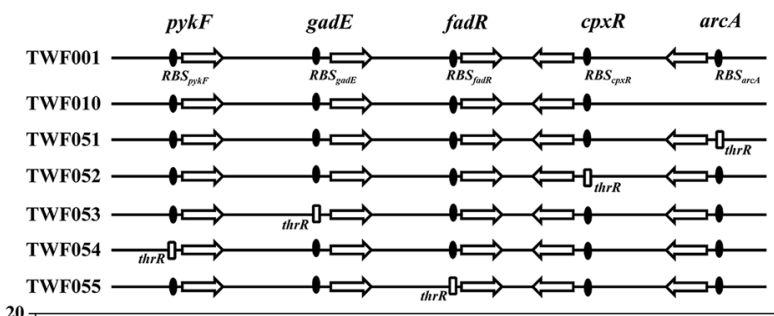

b
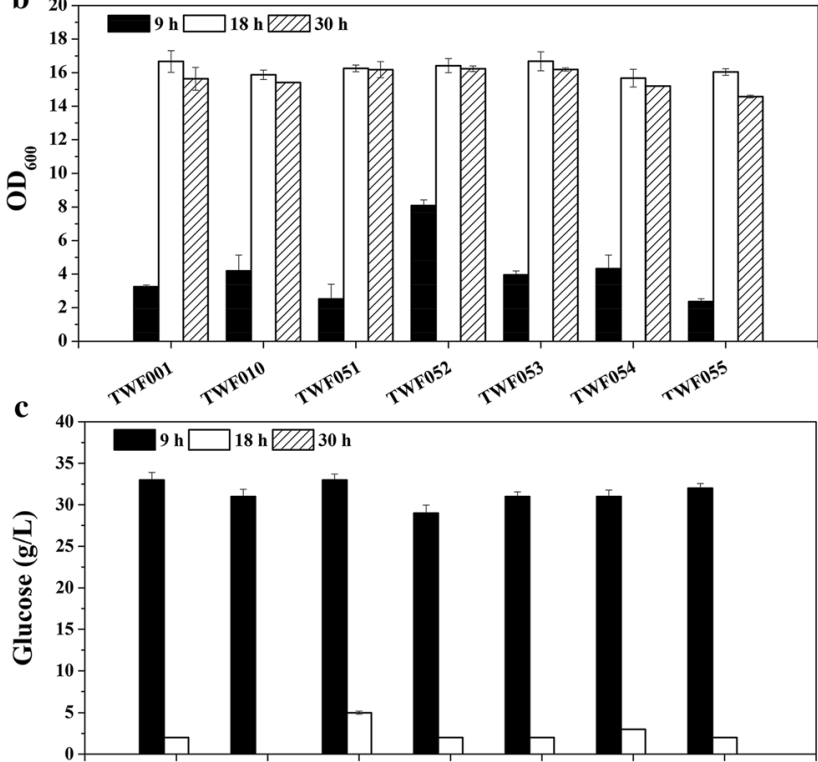

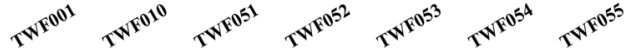
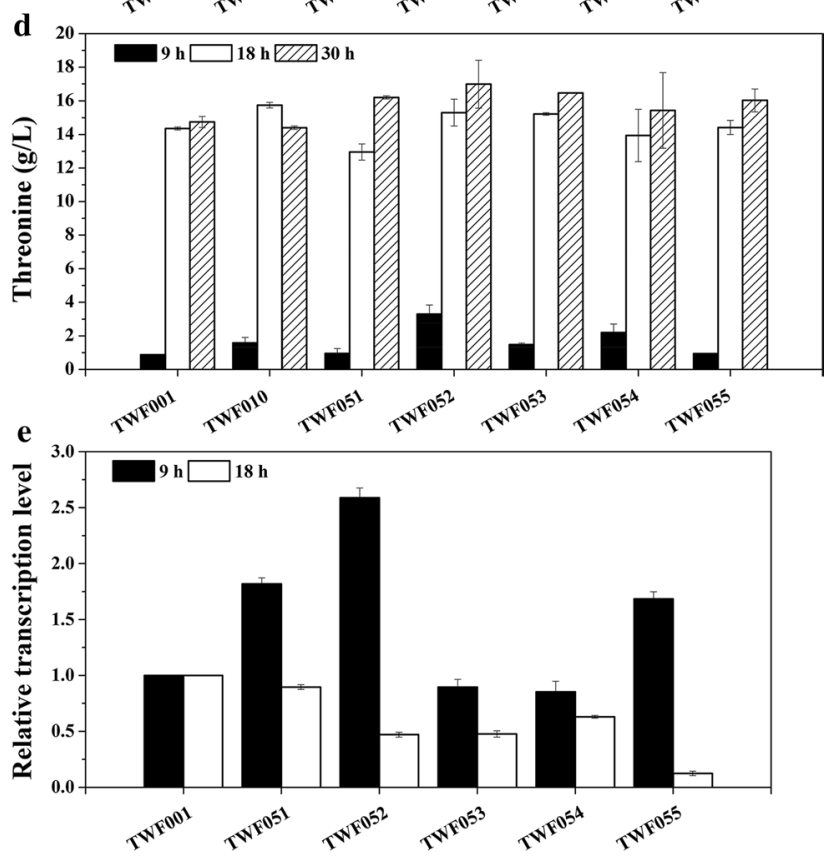

Fig. 2 Flask cultivation for L-threonine production in E. coli strains TWF001, TWF0010, TWF051, TWF052, TWF053, TWF054 and TWF055. a Genetic comparison of the strains constructed by dynamic regulation genes $\operatorname{arcA}, c p x R$, gadE, pykF and fadR. b Cell growth; c glucose consumption; $\mathbf{d} \mathrm{L}$-threonine production; e relative transcription levels of arcA, cpxR, gadE, pykF and fadR in TWF051, TWF052, TWF053, TWF054 and TWF055, respectively. The error bars indicate the standard deviations from three independent experiments 

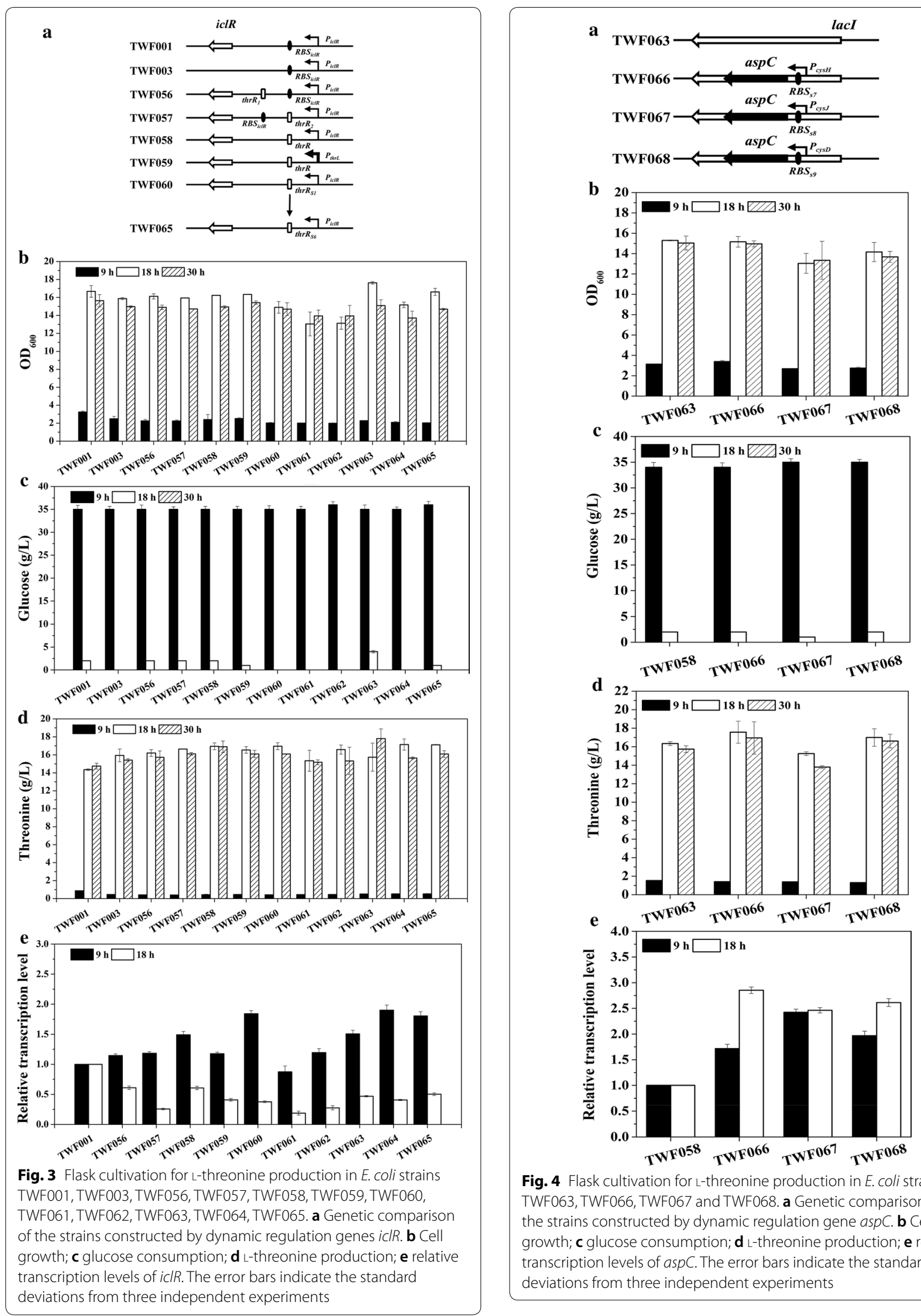

Fig. 4 Flask cultivation for L-threonine production in E. coli strains TWF063, TWF066, TWF067 and TWF068. a Genetic comparison of the strains constructed by dynamic regulation gene aspC. $\mathbf{b}$ Cell growth; $\mathbf{c}$ glucose consumption; $\mathbf{d}$ L-threonine production; e relative transcription levels of aspC. The error bars indicate the standard deviations from three independent experiments 
$\left(P_{c y s}\right)$ and $R B S_{s 8}$ into the lacI locus in chromosome. The fragment of $R B S_{s 8}$-aspC was amplified by using the primers cysJ-aspC-F2/cysH-aspC-R2, and using the genomic DNA of TWF001 as a template. The promoter of $c y s J$ was amplified by using the primers cysJ-aspC-F1/cysJ-aspC$\mathrm{R} 1$, and using the genomic DNA of TWF001 as a template. These two DNA fragments were linked together by overlap PCR, using the primers cys-aspC-F/cys-aspC-R, resulting in the replacement fragment $P_{c y s}-R B S_{s 8}$-asp $C$. TWF068 was constructed from TWF001 by inserting the gene aspC controlled by the promoter of $c y s D\left(P_{c y s D}\right)$ and $R B S_{s 9}$ into the lacI locus in chromosome. The fragment of $R B S_{s 9}$-aspC was amplified by using the primers $c y s D$ aspC-F2/cysH-aspC-R2, and using the genomic DNA of TWF001 as a template. The promoter of $c y s D$ was amplified by using the primers $c y s D$-aspC-F1/cysD-aspC-R1, using the genomic DNA of TWF001 as a template. These two DNA fragments were linked together by overlap $\mathrm{PCR}$, using the primers cys-aspC-F/cys-aspC-R, resulting in the replacement fragment $P_{c y s D}-R B S_{s 9}$-aspC.

As shown in Fig. 5a, TWF069, TWF070, TWF071, TWF072 and TWF073 were derived from TWF066 using the same method to construct TWF051, TWF052, TWF053, TWF054 and TWF055, respectively. TWF074, TWF075, TWF076 and TWF077 were derived from TWF070 using the same method to construct TWF051, TWF053, TWF054 and TWF055, respectively. TWF078, TWF079 and TWF080 were derived from TWF077 using the same method to construct TWF051, TWF053 and TWF054, respectively. TWF081 and TWF082 were derived from TWF078 using the same method to construct TWF053 and TWF054, respectively. TWF083 was derived from TWF081 using the same method to construct TWF054.

\section{Flask fermentation and fed-batch fermentation}

Escherichia coli strains were streaked from the frozen stock on LB plate and cultured at $37^{\circ} \mathrm{C}$ overnight. Then, a loop of bacteria was transferred into a tube containing $5 \mathrm{~mL} \mathrm{LB}$ medium and cultured at $37{ }^{\circ} \mathrm{C}$ for $5 \mathrm{~h}$ with $200 \mathrm{rpm}$ shaking. Next, the culture was transferred into a $250-\mathrm{mL}$ flask containing $30 \mathrm{~mL} \mathrm{LB}$ medium (the initial $\mathrm{OD}_{600}$ of 0.05 ) and cultured at the same growth condition for 6 h. Finally, $5 \mathrm{~mL}$ culture was transferred into a 500$\mathrm{mL}$ flask containing $30 \mathrm{~mL}$ fermentation medium $(2 \mathrm{~g} / \mathrm{L}$ yeast extract, $2 \mathrm{~g} / \mathrm{L}$ citric acid, $25 \mathrm{~g} / \mathrm{L}\left(\mathrm{NH}_{4}\right)_{2} \mathrm{SO}_{4}, 7.46 \mathrm{~g} / \mathrm{L}$ $\mathrm{KH}_{2} \mathrm{PO}_{4}, 40 \mathrm{~g} / \mathrm{L}$ glucose, $2 \mathrm{~g} / \mathrm{L} \mathrm{MgSO}_{4} \cdot 7 \mathrm{H}_{2} \mathrm{O}, 5 \mathrm{mg} / \mathrm{L}$ $\mathrm{FeSO}_{4} \cdot 7 \mathrm{H}_{2} \mathrm{O}, 5 \mathrm{mg} / \mathrm{L} \mathrm{MnSO}_{4} \cdot 4 \mathrm{H}_{2} \mathrm{O}$, and $20 \mathrm{~g} / \mathrm{L} \mathrm{CaCO}_{3}$, $\mathrm{pH}$ 6.8) [50] at $37^{\circ} \mathrm{C}$ with $200 \mathrm{rpm}$ shaking for flask fermentation, or the whole culture was transferred into a quadruple bioreactor (Parallel-Bioreactor, China) containing $1 \mathrm{~L}$ fermentation medium (30 g/L glucose, $20 \mathrm{~g} / \mathrm{L}$ $\left(\mathrm{NH}_{4}\right)_{2} \mathrm{SO}_{4}, 3 \mathrm{~g} / \mathrm{L}$ yeast extract, $2 \mathrm{~g} / \mathrm{L} \mathrm{KH}_{2} \mathrm{PO}_{4}, 2 \mathrm{~g} / \mathrm{L}$
$\mathrm{MgSO}_{4} \cdot 7 \mathrm{H}_{2} \mathrm{O}, 5 \mathrm{mg} / \mathrm{L} \mathrm{FeSO}_{4} \cdot 7 \mathrm{H}_{2} \mathrm{O}, 5 \mathrm{mg} / \mathrm{L}^{-} \mathrm{MnSO}_{4} \cdot 4$ $\mathrm{H}_{2} \mathrm{O}$ ) [6]. Temperature was maintained at $37^{\circ} \mathrm{C}$, the aeration rate at $1.5 \mathrm{vvm}, \mathrm{pH}$ was maintained automatically at 6.9 with $\mathrm{NH}_{4} \mathrm{OH}$, and the dissolved oxygen value was maintained below $30 \%$. Biomass was characterized by the $\mathrm{OD}_{600}$ value. One unit of $\mathrm{OD}_{600}$ corresponds to $1.7 \mathrm{~g} / \mathrm{L}$ cell wet weight [6]. The amount of amino acids was determined by the 1200 series HPLC system (Agilent Technology, USA), using the orthophthalaldehyde precolumn derivatization method [51].

Acetate, pyruvate and oxaloacetate were quantified by using 1200 Series HPLC system (Agilent Technology, USA) equipped with an amines HPX-87H column $(300 \times 7.8 \mathrm{~mm})$, and $0.005 \mathrm{M} \mathrm{H}_{2} \mathrm{SO}_{4}$ was used as a mobile phase with a flow rate of $0.5 \mathrm{~mL} / \mathrm{Min}$. The column temperature was maintained at $40^{\circ}$ orthophthalaldehyde and the UV absorption was determined at $210 \mathrm{~nm}$.

\section{Quantification of mRNA}

The transcriptional levels of $i c l R, \operatorname{arc} A, c p x R, \operatorname{gad} E, p y k F$, $f a d R$ and $a s p C$ in different $E$. coli strains was quantified using the real-time PCR (RT-PCR). Total RNA was extracted from different $E$. coli cells grown at $9 \mathrm{~h}$ and $18 \mathrm{~h}$. According to the published method [52, 53], the relative abundance of the targeted mRNAs was quantified based on the cycle threshold value, which is defined as the number of cycles required to obtain a fluorescent signal above the background and was calculated. The relative abundance of 16S rRNA was used as an internal standard. All assays were performed in triplicate.

\section{Statistical analysis}

Experimental data were expressed as means \pm standard deviations. Statistical comparisons were made by oneway ANOVA to detect significant difference for L-threonine production and glucose consumption between control and experimental groups. Spots with an adjusted p-value $<0.05$ were considered statistically significant.

\section{Results \\ Expression regulation of $\operatorname{arc} A, c p x R, g a d E, p y k F$ and fadR to increase L-threonine production in E. coli TWF001}

The $t h r$ operon controls the expression of $\mathrm{L}$-threonine synthesis in E. coli by thrL [11]. ArcA represses some genes involved in TCA cycle and glyoxylate shunt [34], and $\mathrm{L}$-threonine production increased in the $\operatorname{arcA}$ mutant TWF010 [46]. Pyruvate kinase encoded by $p y k F$ is important for cellular metabolism and glycolysis in $E$. coli, and the $p y k F$ mutant THRD produced more L-threonine [48]. FadR regulates the transcription of most genes involving in fatty acid degradation [28], and the mutant 
a

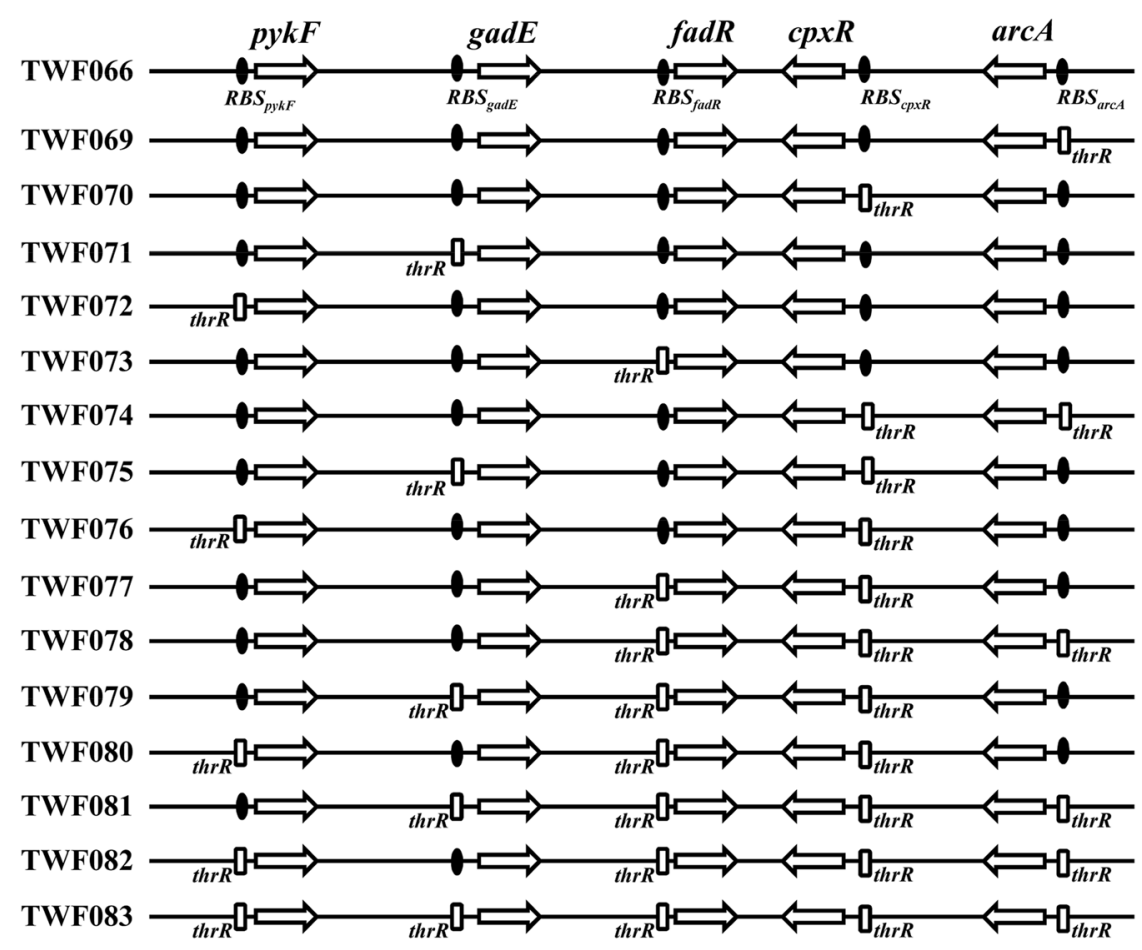

b
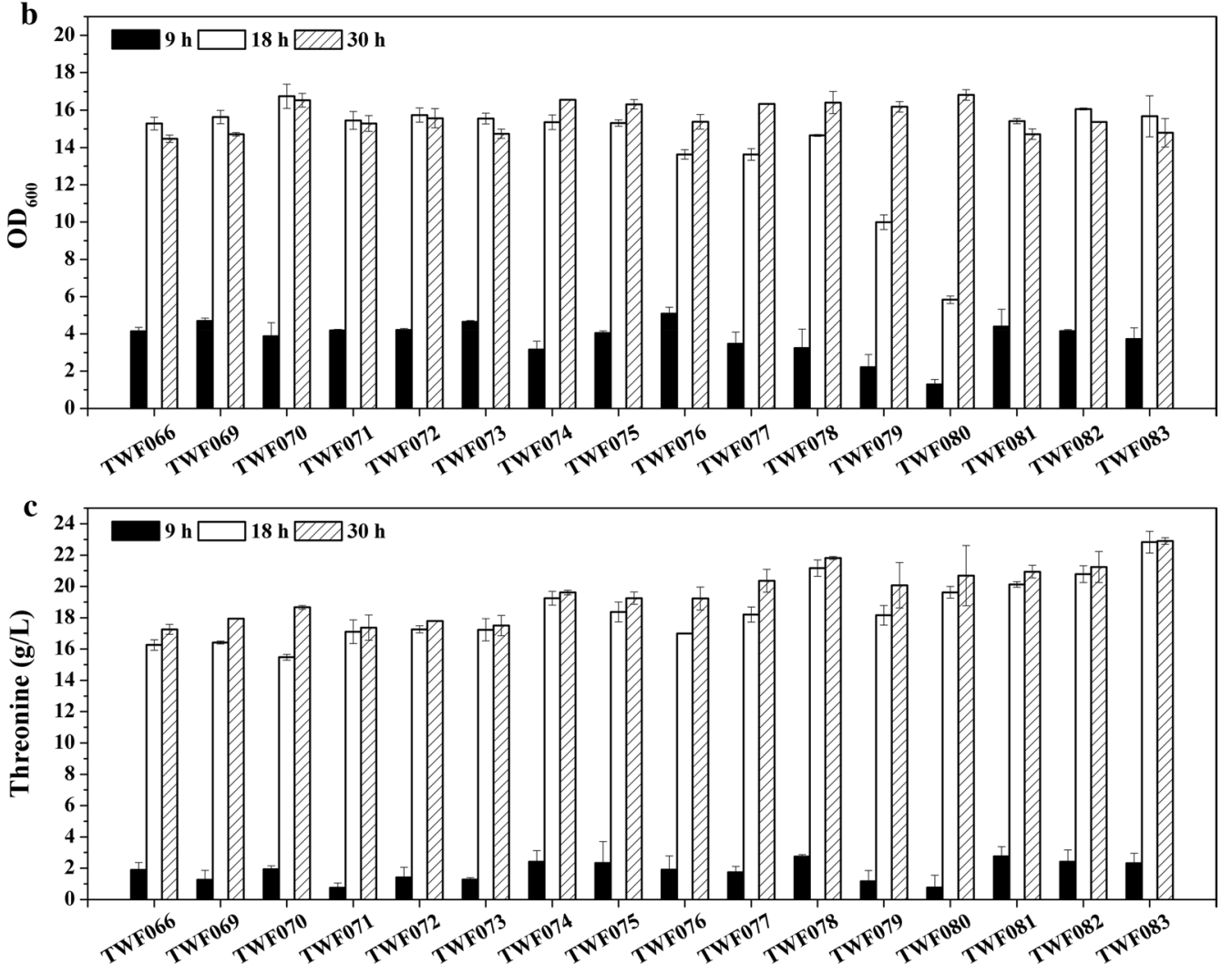

Fig. 5 Flask cultivation for L-threonine production in E. coli strains TWF066, TWF069, TWF070, TWF071, TWF072, TWF073, TWF074, TWF075, TWF076, TWF077, TWF078, TWF079, TWF080, TWF081, TWF082 and TWF083. a Genetic comparison of the strains constructed by dynamic regulation genes $\operatorname{arcA}, c p x R, g a d E, p y k F$ and fadR. $\mathbf{b}$ Cell growth; $\mathbf{c}$--threonine production. The error bars indicate the standard deviations from three independent experiments 
TWF044 in which both $i c l R$ and $f a d R$ were deleted could increase L-threonine production [6]. CpxR and GadE can activate the genes involved in the type II fatty acid synthase systems [32, 33]. Since the genes $\operatorname{arcA}, c p x R, \operatorname{gadE}$, $p y k F$ and $f a d R$ are important for L-threonine biosynthesis in $E$. coli, it is necessary to dynamically regulate their expression. In this study, the RBS sequence of the five genes $\operatorname{arc} A, c p x R, \operatorname{gadE}, p y k F$ and $\operatorname{fad} R$ in TWF001 was individually replaced with $t h r R$, resulting in the stains TWF051, TWF052, TWF053, TWF054 and TWF055, respectively (Fig. 2a).

After $30 \mathrm{~h}$ flask fermentation, the maximum $\mathrm{OD}_{600}$ of E. coli TWF001 reached 16.67 and produced $14.75 \mathrm{~g} / \mathrm{L}$ L-threonine from $40 \mathrm{~g} / \mathrm{L}$ glucose (Fig. 2). Compared with TWF001, the maximum $\mathrm{OD}_{600}$ (15.88) of $\operatorname{arcA}$ deletion mutant TWF010 [46] decreased, but the maximum L-threonine production of TWF010 increased (Fig. 2). TWF010 consumed glucose faster than TWF001 and other five mutant strains, and used up all the glucose after $18 \mathrm{~h}$; after $30 \mathrm{~h}$, glucose was completely consumed in all strains (Fig. 2c).

The maximum L-threonine productions in TWF051, TWF052, TWF053, TWF054 and TWF055 were all lager than TWF001 and TWF010 (Fig. 2d). This indicates that the expression regulation of these five genes is beneficial for L-threonine biosynthesis in E. coli.

The transcriptional levels of $\operatorname{arcA}$ in TWF051, $p p x R$ in TWF052, gadE in TWF053, pykF in TWF054, and fadR in TWF055 were determined (Fig. 2e). All these genes were up-regulated in the early stage of fermentation $(9 \mathrm{~h})$, but down-regulated in the later stage $(18 \mathrm{~h})$. This indicates that the dynamic regulation of the target gene worked in all five mutants and explains the higher L-threonine production in these mutants, although the degree of regulation varies with the target gene. The maximum L-threonine production $(16.99 \mathrm{~g} / \mathrm{L})$ was obtained in TWF052 with a yield of $0.424 \mathrm{~g} / \mathrm{g}$ glucose.

\section{Expression regulation of $i c l R$ to increase L-threonine production in E. coli TWF001}

In previous work, we have developed an L-threonineproducing strain TWF003 from E. coli TWF001 by deleting $i c l R$. The $\mathrm{L}$-threonine production in TWF003 reached $11.76 \mathrm{~g} / \mathrm{L}$, which is a $26 \%$ increase compared to TWF001 [9]. Deleting $f a d R$ [6] or $\operatorname{arcA}$ [46] in TWF003 could further increase L-threonine production. Since IclR regulates the expression of the aceBAK operon which could induce the glyoxylate bypass, and this could bypass prevent the quantitative loss of the entering carbon as $\mathrm{CO}_{2}$ in the Krebs cycle [54], the deletion of iclR might influence the cell growth of $E$. coli; therefore, dynamically regulating the expression of iclR should be a good strategy to balance the cell growth and L-threonine production in TWF001. Since IclR also regulates its own expression, we tried to change its regulatory region to regulate the expression of $i c l R$ according to the intracellular L-threonine concentration. Starting from TWF001, TWF056 was constructed by inserting $t h r R_{1}$ between $R B S_{i c l R}$ and $i c l R$, TWF057 was constructed by inserting $t h r R_{2}$ between $P_{i c l R}$ and $R B S_{i c l R}$, TWF058 was constructed by replacing $R B S_{i c l R}$ with $t h r R$, and TWF059 was constructed by replacing $P_{i c l R}$ and $R B S_{i c l R}$ with $P_{t h r l}$ thrR (Fig. 3a).

During flask fermentation, the maximum $\mathrm{OD}_{600}$ of TWF056, TWF057, TWF058 and TWF059 reached $16.12,15.94,16.24$ and 16.34 , respectively, which are higher than that of TWF001 but lower than that of TWF003 [9] (Fig. 3b). The results indicate that the autoregulation of $i c l R$ expression in these mutants did not significantly affect the cell growth.

Glucose consumption patterns are quite different in these strains. Similar levels of glucose were consumed in these strains after $9 \mathrm{~h}$. After $18 \mathrm{~h}$ glucose was completely consumed in TWF003, TWF060, TWF061, TWF062, and TWF064. After $30 \mathrm{~h}$, glucose was used up in all strains (Fig. 3c). During flask fermentation, TWF056, TWF057, TWF058 and TWF059 produced more L-threonine than TWF001 and TWF003 (Fig. 3d). The results indicate that dynamic regulation of $i c l R$ expression is better than deleting iclR to improve L-threonine biosynthesis in E. coli. Among these four strains, TWF058 produced the highest amount of L-threonine $(16.95 \mathrm{~g} / \mathrm{L})$ after $18 \mathrm{~h}$ flask cultivation, which is $6.34 \%$ more than that of TWF003. This suggests that the dynamic regulatory $i c l R$ expression by replacing $R B S_{i c l R}$ with $t h r R$ is a good choice for improving L-threonine production in E. coli.

To understand the difference on growth rate and L-threonine productions in the above mutant strains, the transcriptional level of $i c l R$ were determined by RTPCR (Fig. 3e). Compared to the control TWF001, the transcriptional level of $i c l R$ in all four mutant strains increased after $9 \mathrm{~h}$ but decreased after $18 \mathrm{~h}$. This suggests that the expression of $i c l R$ in these strains were dynamically regulated, i.e., the expression of $i c l R$ was up-regulated in the early stage of the growth but down-regulated in the late stage of the growth.

In order to optimize the dynamic regulation of iclR expression, different RBS in thrR were designed (Table 4). The mutants TWF060, TWF061, TWF062, TWF063, TWF064 and TWF065 were constructed from TWF058 by replacing $R B S_{i c l R}$ with $t h r R_{s 1}, t h r R_{s 2}, t h r R_{s 3}, t h r R_{s 4}$, $t h r R_{s 5}$, and $t h r R_{s 6}$, respectively (Table 1 and Fig. 3a). After $30 \mathrm{~h}$ cultivation, the maximum $\mathrm{OD}_{600}$ of TWF060, TWF061, TWF062, TWF063, TWF064 and TWF065 reached 14.90, 13.95, 13.95, 17.62, 15.17 and 16.62, respectively; the maximum production of L-threonine in TWF060, TWF061, TWF062, TWF063, TWF064 and 
TWF065 reached 16.96, 15.34, 16.58, 17.81, 17.15 and 17.12, respectively. Among these mutants, TWF063is the best according to the growth rate and L-threonine productions.

The transcriptional levels of iclR in TWF060, TWF061, TWF062, TWF063, TWF064 and TWF065 were also determined by RT-PCR, using TWF001 as the control. Compared to TWF001, the transcription levels of $i c l R$ in TWF061, TWF062, TWF063, TWF064 and TWF065 were up-regulated in the early stage of the growth $(9 \mathrm{~h})$ but down-regulated in the late stage $(18 \mathrm{~h})$. This indicates again that the regulation of $i c l R$ in these mutants satisfies the growth needs in the early stage of fermentation as well as the needs for L-threonine production in the late stage of fermentation. The maximum L-threonine production $(17.81 \mathrm{~g} / \mathrm{L})$ was obtained in TWF063 with a yield of $0.445 \mathrm{~g} / \mathrm{g}$ glucose.

\section{Expression regulation of $\operatorname{aspC}$ to improve L-threonine production in E. coli TWF063}

Previously, L-threonine producing E. coli strain TWF006 was constructed from TWF004 by inserting the trc promoter in the upstream of $a s p C$ in chromosome [9]. The L-threonine production in TWF006 reached $12.47 \mathrm{~g} / \mathrm{L}$ from $30 \mathrm{~g} / \mathrm{L}$ glucose after $36 \mathrm{~h}$ flask fermentation, which is $11.6 \%$ increase compared to the control TWF004 [9]. More L-threonine was also produced when $P_{\text {tac }}$-asp $C$ was inserted into the lac $Z$ locus of $E$. coli TWF041 [6]. Since the expression regulation of $a s p C$ is related to the cell morphology and growth [55], the dynamic expression regulation of $a s p C$ is necessary to improve L-threonine production in $E$. coli. Therefore, the promoters of $c y s H$, cysJ and cysD which can be activated by L-threonine [12] were fused with different RBS sequences to dynamically regulate the $\mathrm{L}$-threonine production in TWF063. The fragments $P_{c y s H^{-}} R B S_{s T^{-}}$asp C, $P_{c y s}-R B S_{s 8^{-}}-a s p C$ or $P_{c y s D^{-}}$ $R B S_{s 9^{-}}$aspC were individually inserted into the lacI loci in TWF063, resulting in strains TWF066, TWF067 and TWF068, respectively (Fig. 4a).

Glucose consumption patterns are quite similar in these strains. After $30 \mathrm{~h}$, glucose was used up in all strains (Fig. 4c). After $30 \mathrm{~h}$ flask fermentation, TWF063 produced $16.34 \mathrm{~g} / \mathrm{L}$ L-threonine from $40 \mathrm{~g} / \mathrm{L}$ glucose and its maximum $\mathrm{OD}_{600}$ reached 15.30 with glucose being completely consumed (Fig. 4). TWF066 grew (15.16) similar with TWF063 but produced more L-threonine $(17.56 \mathrm{~g} / \mathrm{L})$ than TWF063. TWF067 and TWF068 grew slower than TWF063, and TWF067 produced less L-threonine $(15.25 \mathrm{~g} / \mathrm{L})$ than TWF063, but TWF068 produced more L-threonine $(17.00 \mathrm{~g} / \mathrm{L})$ than TWF063. The results indicate that the regulation of these three promoters was different, and different expression of aspC could affect the growth and L-threonine production. To understand the difference on growth and L-threonine productions in the above mutant strains, the transcriptional level of apsC was determined by RT-PCR (Fig. 4e). Compared to the control TWF063, the transcriptional level of aspC was increased in all three mutant strains during fermentation. At $9 \mathrm{~h}$, the expressions of $a s p C$ in TWF066 and TWF068 were lower than that in TWF067, while at $18 \mathrm{~h}$, the expression of aspC in TWF066 and TWF068 was higher than that in TWF067. Among the three mutants, the expression difference of $a s p C$ between $9 \mathrm{~h}$ and $18 \mathrm{~h}$ in TWF066 was the largest, which is consistent with the highest production of L-threonine in TWF066 This suggests that the promoter of $c y s H$ is most sensitive to $\mathrm{L}$-threonine among the three promoters. The maximum L-threonine production $(17.56 \mathrm{~g} / \mathrm{L})$ was obtained in TWF066 with a yield of $0.439 \mathrm{~g} / \mathrm{g}$ glucose.

\section{Expression of multiple genes to improve L-threonine production in E. coli}

Since dynamic regulation of $\operatorname{arcA}, c p x R, \operatorname{gadE}, f a d R$ or pykF could increase $\mathrm{L}$-threonine production in TWF001 (Fig. 2), these genes were also designed to be dynamically regulated in TWF066, resulting in strains TWF069, TWF070, TWF071, TWF072 and TWF073 (Fig. 5a).

After $30 \mathrm{~h}$ flask fermentation, TWF066 can produce $17.35 \mathrm{~g} / \mathrm{L} \mathrm{L}$-threonine from $40 \mathrm{~g} / \mathrm{L}$ glucose and its maximum $\mathrm{OD}_{600}$ reached 15.29 with glucose being completely consumed. The maximum $\mathrm{OD}_{600}$ of TWF069, TWF070, TWF071, TWF072 and TWF073 reached 15.63, 16.75, 15.46, 15.74 and 15.55, respectively. TWF069, TWF070, TWF071, TWF072 and TWF073 produced 17.94, 18.66, 17.37, 17.78 and $17.51 \mathrm{~g} / \mathrm{L}$ L-threonine, respectively. Among the five mutants, TWF070 is the best based on cells growth and L-threonine yield (Fig. 5).

Mutant strains TWF074 (regulating $\operatorname{arcA}$ ), TWF075 (regulating gadE), TWF076 (regulating $p y k F$ ) and TWF077 (regulating $f a d R$ ) was constructed from TWF070 (Fig. 5a). The maximum $\mathrm{OD}_{600}$ of TWF074, TWF075, TWF076 and TWF077 reached 16.56, 16.32, 15.38 and 16.34, respectively. TWF074, TWF075, TWF076 and TWF077 produced 19.61, 19.25, 19.23 and $20.36 \mathrm{~g} / \mathrm{L} \mathrm{L}$-threonine, respectively. Among the four mutants, TWF077 produced the highest L-threonine yield.

Mutant strains TWF078 (regulating $\operatorname{arcA}$ ), TWF079 (regulating gadE) and TWF080 (regulating $p y k F$ ) was constructed from TWF077. The maximum $\mathrm{OD}_{600}$ of TWF078, TWF079 and TWF080 reached 16.41, 16.19 and 16.82, respectively (Fig. 5b). TWF078 and TWF080 produced more L-threonine (21.81 and $20.70 \mathrm{~g} / \mathrm{L}$, respectively) than TWF077. 
From TWF078, mutant strains TWF081 (regulating gadE) and TWF082 (regulating pykF) was constructed. From TWF081, mutant strain TWF083 (regulating $p y k F$ ) was further constructed (Fig. 5a). The maximum $\mathrm{OD}_{600}$ of TWF081, TWF082 and TWF083 reached 15.42, 16.07 and 15.68, respectively. TWF081 and TWF082 produced less L-threonine (20.94 and $21.24 \mathrm{~g} / \mathrm{L}$, respectively) than TWF078, but TWF083 produced more L-threonine $(22.90 \mathrm{~g} / \mathrm{L})$ than TWF078. Among all the mutants, TWF083 produced the highest L-threonine yield.

\section{Comparison of some key L-threonine producing $E$. coli strains}

To compare their high L-threonine production, flask fermentations of some key mutant strains were performed (Fig. 6). Compared with the control TWF001 [56], all mutant strains produced more L-threonine, and TWF083 produced the most (Fig. 6b). The highest specific growth rate was observed around $3 \mathrm{~h}$ for all strains, and then the specific growth rate decreased with time (Fig. 6a). The highest specific L-threonine production rate was observed between 9 and $15 \mathrm{~h}$ in these strains (Fig. 6b). During $6 \mathrm{~h}$ to $15 \mathrm{~h}$, the specific L-threonine production rate of TWF078 was higher than TWF083, but the final production of L-threonine was lower than TWF083; possibly because that the L-threonine production of TWF078 $(0.774 \mathrm{~g} / \mathrm{L})$ was much lower than that of TWF083 $(0.813 \mathrm{~g} / \mathrm{L})$ at $3 \mathrm{~h}$. The acetate concentration in the mutant strains during the fermentation was quite different from that of TWF001, and much less acetate was accumulated in TWF083 (Fig. 6c). The pyruvate concentration in the mutant strains during the fermentation was also quite different from that of TWF001 (Fig. 6d). TWF083 and TWF078 produced more pyruvate than other strains during 6-24 h. This data indicates that in the mutant strains, especially TWF083, more carbon source was used for L-threonine production. TWF083 and TWF078 also produced more oxaloacetate, the precursor for L-threonine synthesis, than other strains during the fermentation (Fig. 6e). The intracellular NADPH content of TWF078 and TWF083 were lower than that of other strains before $18 \mathrm{~h}$, suggesting that they consumed more NADPH for L-threonine biosynthesis (Fig. 6f).

\section{Effect of glucose concentration on L-threonine production in E. coli TWF083}

TWF001 and TWF083 were grown in medium with different glucose concentrations (Fig. 7). When the initial glucose concentration in the medium increased from 30 to $50 \mathrm{~g} / \mathrm{L}$, the cell growth pattern of TWF001 changed. when grown in medium containing 30,40 , and $50 \mathrm{~g} / \mathrm{L}$ initial glucose, glucose in TWF001 was completely consumed after 15,24 and $30 \mathrm{~h}$, and the maximum $\mathrm{OD}_{600}$ of TWF001 reached 13.80, 14.76, and 13.96, respectively (Fig. $7 \mathrm{a}-\mathrm{c}$ ). The cell growth and glucose consumption patterns of TWF083 were quite different from TWF001 (Fig. 7). When the initial glucose was $30 \mathrm{~g} / \mathrm{L}$, the glucose was depleted after $18 \mathrm{~h}$, and the highest $\mathrm{OD}_{600}$ reached 15.44. When the initial glucose was $40 \mathrm{~g} / \mathrm{L}$, the glucose was depleted after $21 \mathrm{~h}$, and the highest $\mathrm{OD}_{600}$ reached 17.44 When the initial glucose was $50 \mathrm{~g} / \mathrm{L}$, the glucose was depleted after $21 \mathrm{~h}$, and the highest $\mathrm{OD}_{600}$ reached 16.36).

L-Threonine productions in TWF001 and TWF083 grown in medium containing with different concentrations of glucose are also shown in Fig. 7. Under the same growth conditions, TWF083 always produced more L-threonine than TWF001. The highest L-threonine production was obtained in either TWF001 or TWF083 when grown in medium containing $40 \mathrm{~g} / \mathrm{L}$ initial glucose. When grown in medium with $30 \mathrm{~g} / \mathrm{L}$ initial glucose for $18 \mathrm{~h}$, TWF083 produced $18.76 \mathrm{~g} / \mathrm{L}$ L-threonine, while TWF001 produced $15.86 \mathrm{~g} / \mathrm{L}$ L-threonine. When grown in medium with $40 \mathrm{~g} / \mathrm{L}$ initial glucose for $21 \mathrm{~h}$, TWF083 produced $26.50 \mathrm{~g} / \mathrm{L}$ L-threonine, and the conversion rate of glucose to $\mathrm{L}$-threonine reached $0.66 \mathrm{~g} / \mathrm{g}$, while TWF001 produced $17.74 \mathrm{~g} / \mathrm{L} \mathrm{L}$-threonine, and the conversion rate of glucose to $\mathrm{L}$-threonine reached $0.44 \mathrm{~g} / \mathrm{g}$. When grown in medium with $50 \mathrm{~g} / \mathrm{L}$ initial glucose for $27 \mathrm{~h}$, TWF083 produced $26.93 \mathrm{~g} / \mathrm{L}$ L-threonine, while TWF001 produced $16.58 \mathrm{~g} / \mathrm{L} \mathrm{L}$-threonine. The results suggest that TWF083 could efficiently consume glucose to produce $\mathrm{L}$-threonine.

When TWF083 grown in the medium containing $40 \mathrm{~g} / \mathrm{L}$ glucose for $21 \mathrm{~h}$, glucose was depleted, and L-threonine production reached the highest (Fig. 7b). Therefore, the fermentation of TWF083 was conducted in medium with a second addition of glucose, using TWF001 as a control (Fig. 7). TWF001 and TWF083 cells were grown in medium with $30 \mathrm{~g} / \mathrm{L}$ initial glucose, and $30 \mathrm{~g} / \mathrm{L}$ glucose was added when the initial glucose was depleted at 15 and $18 \mathrm{~h}$, respectively. The highest L-threonine production in TWF001 and TWF083 reached 19.67 and $23.11 \mathrm{~g} / \mathrm{L}$, respectively (Fig. 7d). TWF001 and TWF083 cells were grown in medium with $40 \mathrm{~g} / \mathrm{L}$ initial glucose, and $40 \mathrm{~g} / \mathrm{L}$ glucose was added when the initial glucose was depleted at $18 \mathrm{~h}$. The highest L-threonine production in TWF001 and TWF083 reached 18.90 and $29.73 \mathrm{~g} / \mathrm{L}$, respectively (Fig. 7e). TWF001 and TWF083 cells were grown in medium with $50 \mathrm{~g} / \mathrm{L}$ initial glucose, and $30 \mathrm{~g} / \mathrm{L}$ glucose was added when the initial glucose was depleted at $21 \mathrm{~h}$. The highest L-threonine production in TWF001 and TWF083 reached 18.72 and $7.33 \mathrm{~g} / \mathrm{L}$, respectively (Fig. 7f). Compared with the control TWF001, TWF083 grown better and produced more L-threonine under the same growth condition. 

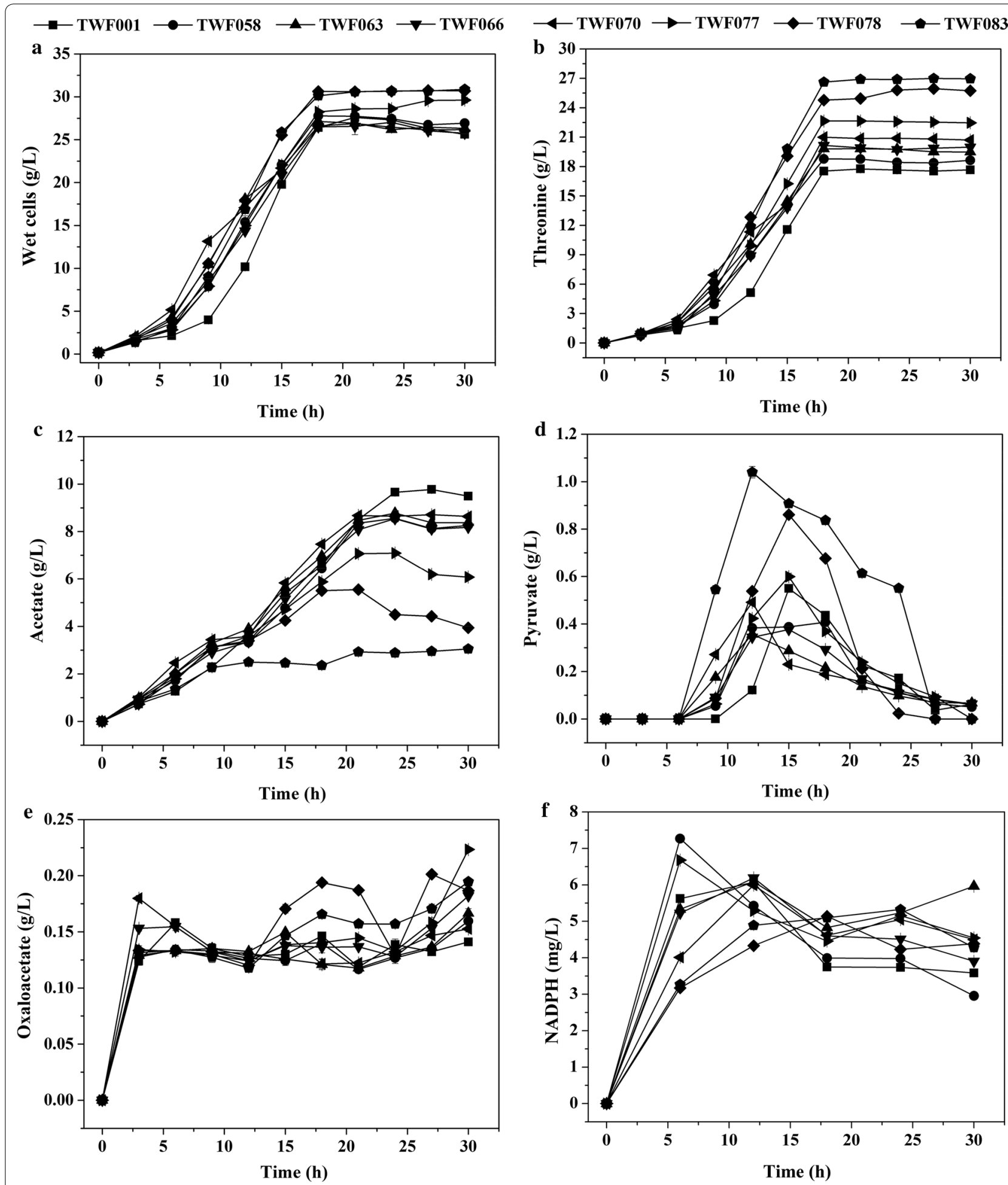

Fig. 6 Comparison of cell growth (a), L-threonine production (b), acetate concentration (c), pyruvate concentration (d), oxaloacetate concentration (e), and NADPH level (f) during flask fermentation of TWF001, TWF058, TWF063, TWF066, TWF070, TWF077, TWF078 and TWF083. The error bars indicate the standard deviations from three independent experiments 


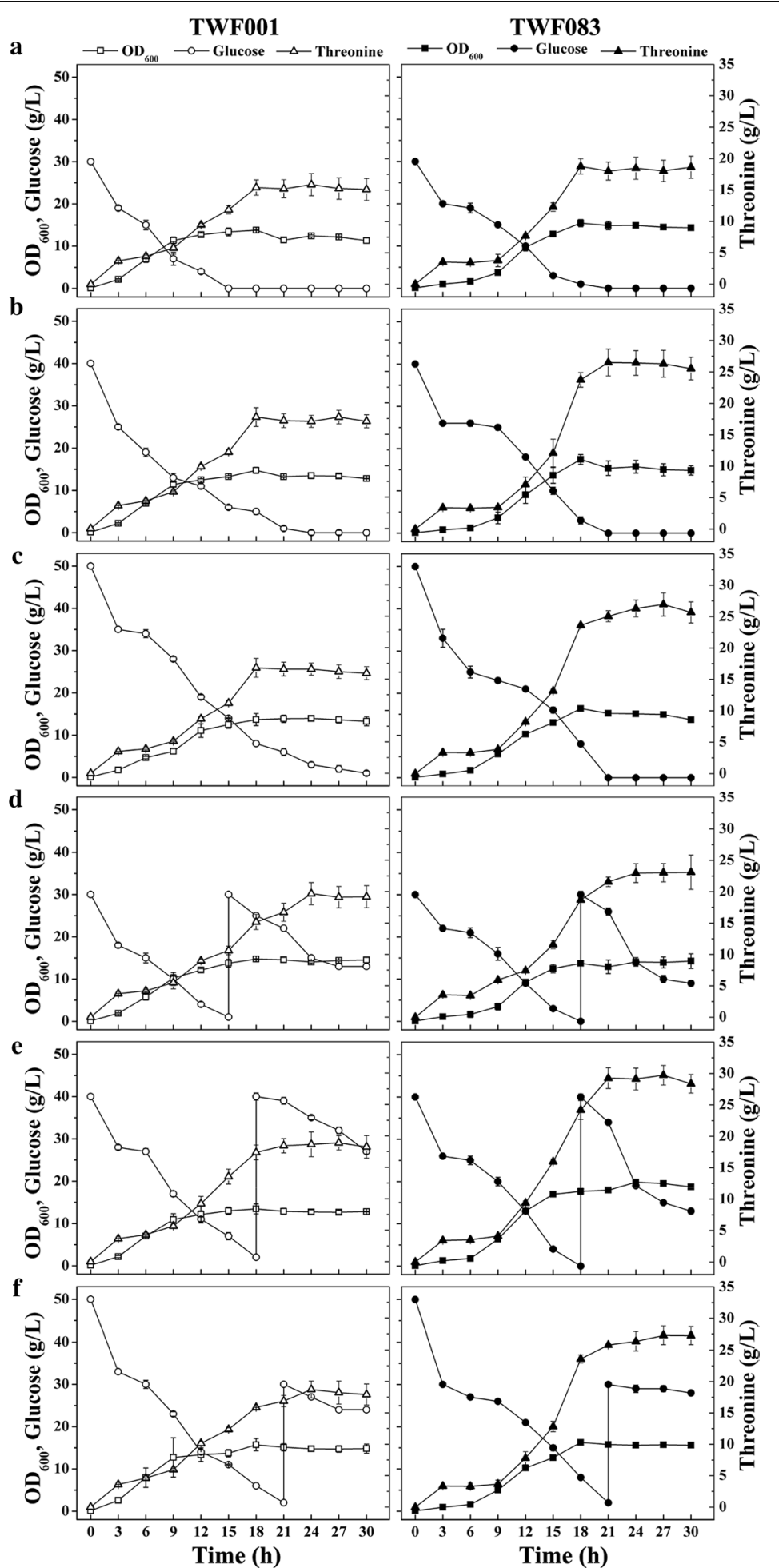

Fig. 7 Comparison for L-threonine production in E. coli strains TWF001 and TWF083 under different glucose addition. a $30 \mathrm{~g} / \mathrm{L}$ glucose; b $40 \mathrm{~g} / \mathrm{L}$ glucose; $\mathbf{c} 50 \mathrm{~g} / \mathrm{L}$ glucose; $\mathbf{d} 2 * 30 \mathrm{~g} / \mathrm{L}$ glucose, the first was added the beginning and the second when the glucose was used up completely, respectively; e $2 * 40 \mathrm{~g} / \mathrm{L}$ glucose, the first was added at the beginning and the second at $18 \mathrm{~h}$, respectively; $\mathbf{f} 50$ and $30 \mathrm{~g} / \mathrm{L}$ glucose, the first was added at the beginning and the second at $21 \mathrm{~h}$, respectively. The error bars indicate the standard deviations from three independent experiments 

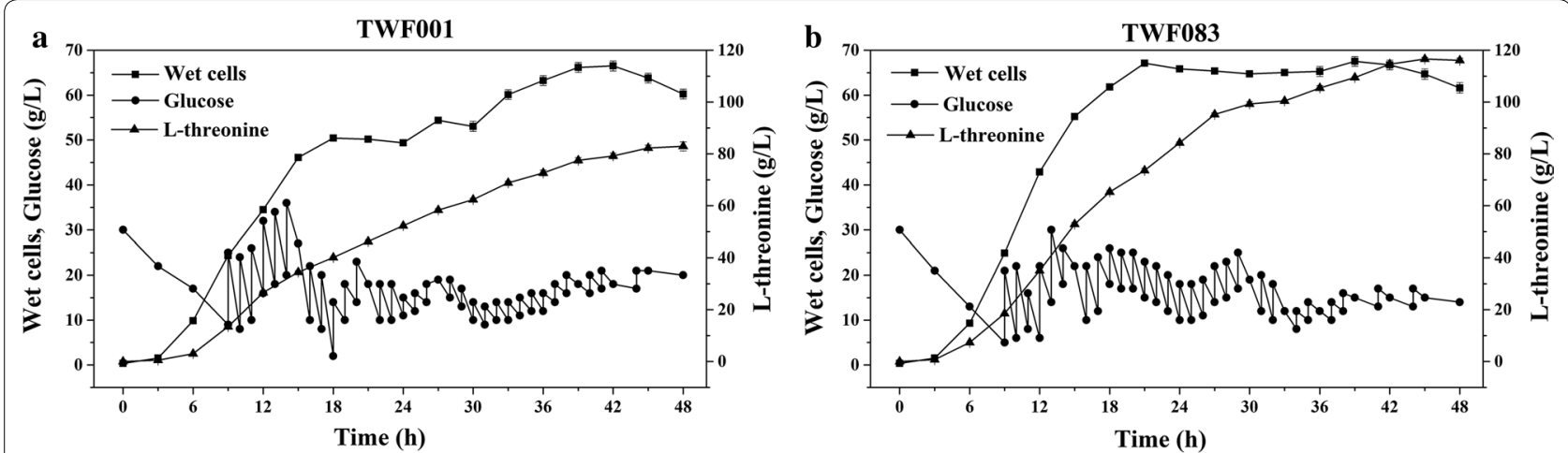

Fig. 8 Fed-batch fermentation of TWF083, using TWF001 as a control. The error bars indicate the standard deviations from three independent experiments

Fed-batch fermentation of TWF083 was performed in 2.4- $\mathrm{L}$ quadruple fermenters with a working volume of $1 \mathrm{~L}$, using TWF001 as a control (Fig. 8). TWF001 could produce $82.9 \mathrm{~g} / \mathrm{L} \mathrm{L}$-threonine in $48 \mathrm{~h}$, leading to a yield of $0.357 \mathrm{~g} / \mathrm{g}$ glucose and a volumetric productivity of $1.73 \mathrm{~g} / \mathrm{L} / \mathrm{h}$. TWF083 produced $116.62 \mathrm{~g} / \mathrm{L}$ L-threonine with a yield of $0.486 \mathrm{~g} / \mathrm{g}$ glucose and productivity of $2.43 \mathrm{~g} / \mathrm{L} / \mathrm{h}$. Both biomass accumulation and glucose consumption of TWF083 were faster than TWF001 in the fermenter, suggesting that the dynamic regulation expression of multiple key genes in TWF083 functions well in large-scale fermentation.

\section{Discussion}

In this study, the thrL regulatory region was used as dynamic regulation element to control the expression of some key genes responsive to L-threonine. The gene $\operatorname{arc} A$ in TCA cycle, the genes $f a d R, c p x R$ and $g a d E$ in fatty acid synthesis and the gene $p y k F$ in aerobic respiration were repressed using the thrR dynamic regulation. The RBSs with different strength in $t h r R$ were designed to optimize the dynamic regulation of the gene $i c l R$ in the glyoxylate shunt. The gene $a s p C$ following same strength RBSs and different threonine-activating promoters, $P_{c y s H}, P_{c y s J}$ and $P_{c y s D}$, were inserted into the chromosome to enhance its expression. Finally, all regulations were integrated into one strain, and the resulting strain TWF083 could produce $26.50 \mathrm{~g}$ L-threonine from $40 \mathrm{~g}$ glucose, which is $49.4 \%$ higher than the control TWF001, and the conversion rate reached $0.66 \mathrm{~g} / \mathrm{g}$ (Fig. $7 \mathrm{~b})$. In recent years, many studies have focused on designing genetic circuits to realize dynamic regulation of metabolic flux for producing intermediates [57] such as lycopene [58], myoinositol [59] and L-lysine [60]. A series of threonine-responsive sensors were derived by transforming thrL leader regulatory elements, which were in the negative correlation with L-threonine concentration and has different sensitivity
[61]. However, there was no report on the application of thrL regulation in $\mathrm{L}$-threonine production.

The modification of glyoxylate shunt has been used to overcome acetate overflow and improve the production of acetyl-CoA-derived chemicals by deleting $i c l R$ in E. coli $[9,22-24,26]$. In this study, the strain TWF056, TWF057, TWF058, TWF059, TWF060, TWF061, TWF062, TWF063, TWF064 and TWF065 were constructed by inserting the different part of attenuator region of $t h r$ operon, $t h r L$, into the different native regulatory regions in front of $i c l R$. All these strains could produce more L-threonine than the control TWF001 after $30 \mathrm{~h}$ fermentation. TWF063 produced $11.7 \%$ more L-threonine than the iclR deletion mutant TWF003 [9], and its growth was also improved (Fig. 3).

The aspartate aminotransferase encoded by aspC draws the carbon flux from TCA cycle to the biosynthetic pathway of $\mathrm{L}$-aspartate family. Deletion of $a s p C$ led to generation of small cells with slow growth, while overexpression of $a s p C$ exerted the opposite effect [55]. Using a switch to make the glycolytic fluxes towards TCA cycle in the early stage, and to make the carbon flux be redirected into L-threonine synthetic pathway by inducing the expression of $a s p C, f d h, g d h A$ and pntAB in the production stage could increase L-threonine production [62]. The gene $a s p C$ following different threonine-enhancing promoters were inserted into the chromosome of TWF063, resulting in the strains TWF066, TWF067 and TWF068. Because of the high expression of $a s p C$ in early stage, the growth of TWF067 and TWF068 was inhibited and less L-threonine was produced, but the lower expression of $\operatorname{aspC}$ at the early stage and the higher expression at the later stage made the growth of TWF066 unaffected, but the L-threonine production increased by 7\% (Fig. 4).

The global regulation factor $\operatorname{arc} A$ deletion has been used in E. coli to activate the TCA cycle and aerobic respiration $[46,63]$. The global regulation factor $f a d R$ 
deletion has been used to increase the acetyl-CoA supply by enhancing the fatty acid degradation [6]. The role of $c p \times R$ and gadE in fatty acid synthesis is to activate $f a b A$ and $f a b Z$ [32, 33], which are not regulated by $f a d R$, but there was no report on the accumulation of substances by knocking out $c p x R$ and $g a d E$. In $p y k F$ knockout mutant, less acetate and more L-threonine was produced [47, 48]. With the dynamic regulation, the nature regulatory regions of the above 5 genes were replaced by thrL leader regulatory elements in TWF001, resulting in the strains TWF051, TWF052, TWF053, TWF054 and TWF055. All these mutants could produce more L-threonine than the control TWF001 after $30 \mathrm{~h}$ fermentation. TWF066 was used as a starting strain, step by step, to carry out multiple knockout test on the 5 genes to find the optimal strain. The results showed that the genetic engineering through the dynamic regulation had little effect on the metabolism of the strain in the early stage. After several rounds of modifications, the growth of strain TWF083 was similar to that of original strain TWF001 but the L-threonine production increased by $49.4 \%$.

Several L-threonine producing E. coli strains have been constructed. An L-methionine auxotroph strain KY10935 obtained by a classical selection method without the use of directed genetic-engineering modifications could produce $100 \mathrm{~g} / \mathrm{L}$ L-threonine after $77 \mathrm{~h}$ cultivation [65]. TH28C/pBRThrABCR3 constructed from W3110 by systems metabolic engineering and in silico flux response analysis could produce threonine with a high yield of $0.393 \mathrm{~g} / \mathrm{g}$ glucose and $82.4 \mathrm{~g} / \mathrm{L}$ threonine by fed-batch culture [8]. KCCM 10353 could produce $112 \mathrm{~g} / \mathrm{L}$ L-threonine with a high yield of $0.452 \mathrm{~g} / \mathrm{g}$ glucose after $77 \mathrm{~h}$ cultivation [66]. EC125 could produce $105.3 \mathrm{~g} / \mathrm{L}$ L-threonine with a yield of $0.405 \mathrm{~g} / \mathrm{g}$ glucose after $48 \mathrm{~h}$ two-stage feeding cultivation [67]. THRD $\triangle p y k F$ produced less acetate (52\%) and produced $112.57 \mathrm{~g} / \mathrm{L} \mathrm{L}$-threonine with a yield of $0.376 \mathrm{~g} / \mathrm{g}$ glucose after $40 \mathrm{~h}$ cultivation [48]. TRFC derived by repeated compound mutagenesis (DES plus UV) from E. coli K12 could use sucrose as the carbon source, it could produce $124.57 \mathrm{~g} / \mathrm{L} \mathrm{L}$-threonine after $40 \mathrm{~h}$ cultivation, using the combined feeding strategy of pseudo-exponential feeding and glucose-stat feeding resulted in high cell density [68]. THPE5 uses a switch to make the glycolytic fluxes towards TCA cycle in the early stage, leading to the improved glucose utilization and growth performance; in the production stage the carbon flux is redirected into L-threonine synthetic pathway via a synthetic genetic circuit by inducing the expression of asp $C, f d h, g d h A$ and $p n t A B$ to increase L-threonine production, resulting in $70.5 \mathrm{~g} / \mathrm{L} \mathrm{L}$-threonine with a yield of $0.404 \mathrm{~g} / \mathrm{g}$ glucose after $40 \mathrm{~h}$ cultivation [62]. JLTHR could produce $127.3 \mathrm{~g} / \mathrm{L}$ L-threonine with a glucose conversion rate of $58.12 \%$ after adding betaine hydrochloride in the medium [4]. There are a few L-threonine producing strains were developed from the same origin to TWF083. TWF044 could enhance glyoxylate shunt pathway and produce $103.89 \mathrm{~g} / \mathrm{L}$ L-threonine with a yield of $0.450 \mathrm{~g} / \mathrm{g}$ glucose after $48 \mathrm{~h}$ fed-batch fermentation [6]. TWF006/pFW01-thrA*BC-asd could produce only $15.85 \mathrm{~g} / \mathrm{L}$ L-threonine but showed a high yield of $0.528 \mathrm{~g} / \mathrm{g}$ glucose after $36 \mathrm{~h}$ shake flask cultivation [9]. TWF018 could produce $25.1 \mathrm{~g} / \mathrm{L}$ L-threonine with a high yield of $0.628 \mathrm{~g} / \mathrm{g}$ glucose after $36 \mathrm{~h}$ shake flask cultivation [46]. WMZ016/pFW01-thrA*BC-rhtC derived from MG1655 by modifying the glucose specific phosphotransferase system could produce $17.98 \mathrm{~g} / \mathrm{L}$ L-threonine with a yield of $0.346 \mathrm{~g} / \mathrm{g}$ glucose after $36 \mathrm{~h}$ shake flask cultivation [69]. When TWF083 was cultured in shake flask, it produced $16.50 \mathrm{~g} / \mathrm{L}$ L-threonine with a yield of $0.662 \mathrm{~g} / \mathrm{g}$ glucose after $30 \mathrm{~h}$, which is better than other strains derived from the same origin. After $48 \mathrm{~h}$ fed-batch fermentation, TWF083 could produce $116.62 \mathrm{~g} / \mathrm{L} \mathrm{L}$-threonine with a yield of $0.486 \mathrm{~g} / \mathrm{g}$ glucose.

$\mathrm{NADPH}$ is essential for the catalytic activity of glutamate dehydrogenase in E. coli [70]. It also plays a key role in L-threonine biosynthesis due to the NADPHdependent $\beta$-aspartate semialdehyde dehydrogenase and homoserine dehydrogenase [71, 72]. In E. coli, NADPH is mostly produced in the pentose phosphate pathway by glucose-6-phosphate dehydrogenase encoded by the gene $z w f$, which catalyzes the oxidation of glucose 6-phosphate to 6-phosphoglucono- $\delta$-lactone; 6-phosphogluconate dehydrogenase encoded by the genes gnd could also produce NADPH through the oxidative decarboxylation of 6-phosphogluconate to ribulose 5-phosphate [73]. Several approaches to increase the NADPH yield through metabolic engineering of genes involved in the pentose phosphate pathway have been reported [74-80]. Therefore, it is worth studying the influence of NADPH on L-threonine production in TWF083.

In this study, a range of approaches have been proposed for the construction of L-threonine producing strain using dynamic regulation. The successful application of threonine-responsive elements to improve L-threonine production in this work provides further opportunities for synthetic biology and metabolic engineering in E. coli.

\section{Authors' contributions \\ $L Z, Y L$ and $X W$ designed the experiments. JY, YF, LZ, ZD, CW, WM and XH participated in the discussion about the experiments. LZ performed experi- ments and drafted the manuscript. All authors read and approved the final manuscript.}

\section{Funding}

This study was supported by the National Key Research and Development Program of China (2018YFA0900300) and the National First-class Discipline Program of Light Industry Technology and Engineering (LITE2018-10). 


\section{Availability of data and materials}

All data generated or analyzed during this study are included in the manuscript.

\section{Competing interests}

The authors declare that they have no competing interests.

\section{Author details}

1 State Key Laboratory of Food Science and Technology, Jiangnan University, 1800 Lihu Avenue, Wuxi 214122, China. ${ }^{2}$ International Joint Laboratory on Food Safety, Jiangnan University, Wuxi 214122, China. ${ }^{3}$ Nanjing Customs District P. R. China, Wuxi 214122, China. ${ }^{4}$ Key Laboratory of Industrial Biotechnology, Ministry of Education, School of Biotechnology, Jiangnan University, Wuxi 214122, China.

Received: 9 December 2019 Accepted: 18 February 2020 Published online: 24 February 2020

\section{References}

1. Dong X, Quinn PJ, Wang X. Microbial metabolic engineering for L-threonine production. Subcell Biochem. 2012;64:283-302.

2. Lee M-H, Lee H-W, Park J-H, Ahn J-O, Jung J-K, Hwang Y-I. Improved L-threonine production of Escherichia coli mutant by optimization of culture conditions. J Biosci Bioeng. 2006;101(2):127-30.

3. Dong X, Quinn PJ, Wang X. Metabolic engineering of Escherichia coli and Corynebacterium glutamicum for the production of L-threonine. Biotechnol Adv. 2011;29(1):11-23.

4. Su Y, Guo QQ, Wang S, Zhang X, Wang J. Effects of betaine supplementation on $\mathrm{L}$-threonine fed-batch fermentation by Escherichia coli. Bioprocess Biosyst Eng. 2018;41(10):1509-18.

5. Zhang X, Yan J, Yu L, Zhang G, Zhang Y, Chen N, Wen T. Construction of recombinant plasmids containing threonine operon and their effects on L-threonine accumulation. Acta Microbiol Sin. 2009:49:591.

6. Yang J, Fang Y, Wang J, Wang C, Zhao L, Wang X. Deletion of regulatorencoding genes fadR, fabR and iclR to increase L-threonine production in Escherichia coli. Appl Microbiol Biotechnol. 2019;103:4549-64.

7. Livshits VA, Zakataeva NP, Aleshin W, Vitushkina MV. Identification and characterization of the new gene rhtA involved in threonine and homoserine efflux in Escherichia coli. Res Microbiol. 2003;154:123-35.

8. Lee KH, Park JH, Kim TY, Kim HU, Lee SY. Systems metabolic engineering of Escherichia coli for L-threonine production. Mol Syst Biol. 2007;3:149.

9. Zhao H, Fang Y, Wang X, Zhao L, Wang J, Li Y. Increasing L-threonine production in Escherichia coli by engineering the glyoxylate shunt and the L-threonine biosynthesis pathway. Appl Microbiol Biotechnol. 2018;102:5505-18.

10. Breaker RR. Prospects for riboswitch discovery and analysis. Mol Cell. 2011;43:867-79.

11. Lynn SP, Burton WS, Donohue TJ, Gould RM, Gumport RI, Gardner JF. Specificity of the attenuation response of the threonine operon of Escherichia coli is determined by the threonine and isoleucine codons in the leader transcript. J Mol Biol. 1987;194:59-69.

12. Liu YN, Li Q, Zheng P, Zhang Z, Liu Y, Sun C, Cao G, Zhou W, Wang X, Zhang $D$, et al. Developing a high-throughput screening method for threonine overproduction based on an artificial promoter. Microb Cell Fact. 2015;14:121

13. Mohsin M, Ahmad A. Genetically-encoded nanosensor for quantitative monitoring of methionine in bacterial and yeast cells. Biosens Bioelectron. 2014;59:358-64.

14. Mustafi N, Frunzke J. Development and application of a single-cell biosensor for the detection of L-methionine and branched-chain amino acids. Metab Eng. 2012;14:449-57.

15. Binder S, Schendzielorz G, Stabler N, Krumbach K, Hoffmann K, Bott M, Eggeling L. A high-throughput approach to identify genomic variants of bacterial metabolite producers at the single-cell level. Genome Biol. 2012;13:R40.

16. Hoffmann K, Grunberger A, Lausberg F, Bott M, Eggeling L. Visualization of imbalances in sulfur assimilation and synthesis of sulfur-containing amino acids at the single-cell Level. Appl Environ Microbiol. 2013;79:6730-6.
17. Garcia JR, Cha HJ, Rao G, Marten MR, Bentley WE. Microbialnar-GFP cell sensors reveal oxygen limitations in highly agitated and aerated laboratory-scale fermentors. Microb Cell Fact. 2009;8:6-6.

18. Binder S, Siedler S, Marienhagen J, Bott M, Eggeling L. Recombineering in Corynebacterium glutamicum combined with optical nanosensors: a general strategy for fast producer strain generation. Nucleic Acids Res. 2013;41:6360.

19. Schendzielorz G, Dippong M, Grünberger A, Kohlheyer D, Eggeling L. Taking control over control: use of product sensing in single cells to remove flux control at key enzymes in biosynthesis pathways. ACS Synth Biol. 2014;3:21.

20. Lee JH, Wendisch VF. Production of amino acids-Genetic and metabolic engineering approaches. Bioresour Technol. 2017;245:1575-87.

21. Yamamoto K, Ishihama A. Two different modes of transcription repression of the Escherichia coli acetate operon by IcIR. Mol Microbiol. 2003:47:183-94.

22. Liu M, Ding Y, Chen H, Zhao Z, Liu H, Xian M, Zhao G. Improving the production of acetyl-CoA-derived chemicals in Escherichia coli BL21(DE3) through iclR and arCA deletion. BMC Microbiol. 2017;17:10.

23. Li Y, Huang B, Wu H, Li Z, Ye Q, Zhang YP, Li Y, Huang B, Wu H, Li Z. Production of succinate from acetate by metabolically engineered Escherichia coli. ACS Synth Biol. 2016:5:1299.

24. Zhang T, Wang Z, Deng L, Tan T, Wang F, Yan Y. Pull-in urea cycle for the production of fumaric acid in Escherichia coli. Appl Microbiol Biotechnol. 2015:99:5033-44.

25. Woo SC, In KD, Sol C, Jae Won J, Yup LS. Metabolic engineering of Escherichia coli for the production of fumaric acid. Biotechnol Bioeng. 2013;110:2025-34.

26. Ning Y, Wu X, Zhang C, Xu Q, Chen N, Xie X. Pathway construction and metabolic engineering for fermentative production of ectoine in Escherichia coli. Metab Eng. 2016;36:10-8.

27. Fengming L, Yu C, Robert L, Kilho L, Yingjin Y, Xiaoxia Nina L. Improving fatty acid availability for bio-hydrocarbon production in Escherichia coli by metabolic engineering. PLoS ONE. 2013;8:e78595.

28. Campbell JW, Morgan-Kiss RM, Cronan JE. A new Escherichia coli metabolic competency: growth on fatty acids by a novel anaerobic $\beta$-oxidation pathway. Mol Microbiol. 2010;47:793-805.

29. Campbell JW, Cronan JE. Escherichia coli fadR positively regulates transcription of the $f a b B$ fatty acid biosynthetic gene. J Bacteriol. 2001:183:5982-90.

30. My L, Ghandour Achkar N, Viala JP, Bouveret E, de Boer P. Reassessment of the genetic regulation of fatty acid synthesis in Escherichia coli: global positive control by the dual functional regulator FadR. J Bacteriol. 2015;197:1862-72.

31. My L, Rekoske B, Lemke JJ, Viala JP, Gourse RL, Bouveret E. Transcription of the Escherichia coli fatty acid synthesis operon fabHDG is directly activated by FadR andinhibited by ppGpp. J Bacteriol. 2013;195:3784-95.

32. Hommais F. GadE (YhiE): a novel activator involved in the response to acid environment in Escherichia coli. Microbiology. 2004;150:61.

33. Dartigalongue C, Missiakas D, Raina S. Characterization of the Escherichia coli oE Regulon. J Biol Chem. 2001;276:20866-75.

34. Iuchi $\mathrm{S}$, Lin EC. $\operatorname{arcA}(d y e), \operatorname{arcA}(d y e)$, a global regulatory gene in Escherichia coli mediating repression of enzymes in aerobic pathways. Proc Natl Acad Sci USA. 1988:85:1888-92.

35. Park SJ, McCabe J, Turna J, Gunsalus RP. Regulation of the citrate synthase ( $g / t A)$ gene of Escherichia coli in response to anaerobiosis and carbon supply: role of the arcA gene product. J Bacteriol. 1994;176:5086-92.

36. Cunningham L, Gruer MJ, Guest JR. Transcriptional regulation of the aconitase genes ( $a c n A$ and $a c n B$ ) of Escherichia coli. Microbiology. 1997:143:3795-805.

37. Chao G, Shen J, Tseng CP, Park SJ, Gunsalus RP. Aerobic regulation of isocitrate dehydrogenase gene (icd) expression in Escherichia coli by the $\operatorname{arcA}$ and fnr gene products. J Bacteriol. 1997;179:4299-304.

38. Park SJ, Chao G, Gunsalus RP. Aerobic regulation of the suCABCD genes of Escherichia coli, which encode alpha-ketoglutarate dehydrogenase and succinyl coenzyme A synthetase: roles of ArcA, Fnr, and the upstream sdhCDAB promoter. J Bacteriol. 1997;179:4138-42.

39. Spencer ME, Guest JR. Transcription analysis of the sUCAB, aceEF and Ipd genes of Escherichia coli. Mol Gen Genet. 1985;200:145. 
40. Park SJ. Regulation of succinate dehydrogenase (sdhCDAB) operon expression in Escherichia coli in response to and anaerobiosis: role of ArcA and Fnr. Mol Microbiol. 2010;15:473-82.

41. Park SJ, Gunsalus RP. Oxygen, iron, carbon, and superoxide control of the fumarase fumA and fumC genes of Escherichia coli: role of the $\operatorname{arcA}$, fnr, and soxR gene products. J Bacteriol. 1995;177:6255-62.

42. Tseng CP. Regulation of fumarase (fumB) gene expression in Escherichia coli in response to oxygen, iron and heme availability: role of the $\operatorname{arcA}$, fur and hemA gene products. FEMS Microbiol Lett. 2010;157:67-72.

43. Park SJ, Cotter PA, Gunsalus RP. Regulation of malate dehydrogenase ( $m d h)$ gene expression in Escherichia coli in response to oxygen, carbon, and heme availability. J Bacteriol. 1995;177:6652.

44. Pan B, Unnikrishnan I, Laporte DC. The binding site of the ICIR repressor protein overlaps the promoter of aceBAK. J Bacteriol. 1996;178:3982-4.

45. Quail MA, Haydon DJ, Guest JR. The pdhR-aceEF-Ipd operon of Escherichia coli expresses the pyruvate dehydrogenase complex. Mol Microbiol. 2010;12:95-104.

46. Ding Z, Fang Y, Zhu L, Wang J, Wang X. Deletion of $\operatorname{arcA}$, iclR, and $t d c C$ in Escherichia coli to improve L-threonine production. Biotechnol Appl Biochem. 2019:66:794-807.

47. Siddiquee KA, Arauzo-Bravo MJ, Shimizu K. Effect of a pyruvate kinase (pykF-gene) knockout mutation on the control of gene expression and metabolic fluxes in Escherichia coli. FEMS Microbiol Lett. 2004;235:25-33.

48. Xie XX, Liang Y, Liu H, Liu Y, Xu Q, Zhang C, Chen N. Modification of glycolysis and its effect on the production of L-threonine in Escherichia coli. J Ind Microbiol Biotechnol. 2014;41:1007-15.

49. Jiang Y, Chen B, Duan C, Sun B, Yang J, Yang S. Multigene editing in the Escherichia coli genome via the CRISPR-Cas9 system. Appl Environ Microbiol. 2015;81:2506-14.

50. Lee JH, Sung BH, Kim MS, Blattner FR, Yoon BH, Kim JH, Kim SC. Metabolic engineering of a reduced-genome strain of Escherichia coli for L-threonine production. Microb Cell Fact. 2009;8:2.

51. Körös Á, Varga Z, Molnár-Perl I. Simultaneous analysis of amino acids and amines as their o-phthalaldehyde-ethanethiol-9-fluorenylmethyl chloroformate derivatives in cheese by high-performance liquid chromatography. J Chromatogr A. 2008;1203:146-52.

52. Livak KJ, Schmittgen TD. Analysis of relative gene expression data using real-time quantitative PCR and the 2(-Delta Delta $C(T)$ ) method. Methods. 2001;25:402-8.

53. Nolden L, Farwick M, Krämer R, Burkovski A. Glutamine synthetases of Corynebacterium glutamicum: transcriptional control and regulation of activity. FEMS Microbiol Lett. 2001;201:91-8.

54. Gui L, Sunnarborg A, Pan B, Laporte DC. Autoregulation of iclR, the gene encoding the repressor of the glyoxylate bypass operon. J Bacteriol. 1996;178:321-4

55. Liu F, Qimuge JH, Hao J, Yan H, Bach T, Fan L, Morigen. AspC-mediated aspartate metabolism coordinates the Escherichia coli cell cycle. PLoS ONE. 2014;9:e92229.

56. Zhao L, Zhang H, Wang X, Han G, Ma W, Hu X, Li Y. Transcriptomic analysis of an L-threonine-producing Escherichia coli TWF001. Biotechnol Appl Biochem. 2020. https://doi.org/10.1002/bab.1890.

57. Dahl RH, Zhang F, Alonso-Gutierrez J, Baidoo E, Batth TS, Redding-Johanson AM, Petzold CJ, Mukhopadhyay A, Lee TS, Adams PD, Keasling JD. Engineering dynamic pathway regulation using stress-response promoters. Nat Biotechnol. 2013:31:1039.

58. Farmer W, Liao J. Improving lycopene production in Escherichia coli by engineering metabolic control. Nat Biotechnol. 2000;18:533-7.

59. Gupta A, Reizman IMB, Reisch CR, Prather KLJ. Dynamic regulation of metabolic flux in engineered bacteria using a pathway-independent quorum-sensing circuit. Nat Biotechnol. 2017;35:273.

60. Zhou LB, Zeng AP. Exploring lysine riboswitch for metabolic flux control and improvement of L-lysine synthesis in Corynebacterium glutamicum. ACS Synth Biol. 2015;4:729-34.

61. Liu Z, Zheng $Y$, Chen T, Ma H. Transform thrL leading regulatory elements for different threonine sensor. Biotechnology. 2016;26:372-9.

62. Liu J, Li H, Xiong H, Xie X, Chen N, Zhao G, Caiyin Q, Zhu H, Qiao J. Twostage carbon distribution and cofactor generation for improving L-threonine production of Escherichia coli. Biotechnol Bioeng. 2018;116:110-20.
63. Yao P, Cui Y, Yu S, Du Y, Feng J, Wu Q, Zhu D. Efficient biosynthesis of (R)or (S)-2-hydroxybutyrate fromL-threonine through a synthetic biology approach. Adv Synth Catal. 2016;358:2923-8.

64. Espah Borujeni A, Cetnar D, Farasat I, Smith A, Lundgren N, Salis HM. Precise quantification of translation inhibition by mRNA structures that overlap with the ribosomal footprint in N-terminal coding sequences. Nucleic Acids Res. 2017:45:5437-48.

65. Okamoto K, Kino K, Ikeda M. Hyperproduction of L-threonine by an Escherichia coli mutant with impaired L-threonine uptake. Biosci Biotechnol Biochem. 1997;61:1877-82.

66. Yuzbashev TV, Vybornaya TV, Larina AS, Gvilava IT, Voyushina NE, Mokrova SS, Yuzbasheva EY, Manukhov IV, Sineoky SP, Debabov VG. Directed modification of Escherichia coli metabolism for the design of threonineproducing strains. Appl Biochem Micro. 2013;49:723-42.

67. Liu S, Liang Y, Liu Q, Tao T, Lai S, Chen N, Wen T. Development of a twostage feeding strategy based on the kind and level of feeding nutrients for improving fed-batch production of L-threonine by Escherichia coli. Appl Microbiol Biotechnol. 2013;97:573-83.

68. Wang J, Cheng LK, Chen N. High-level production of L-threonine by recombinant Escherichia coli with combined feeding strategies. Biotechnol Biotechnol Equip. 2014;28:495-501.

69. Zhu L, Fang Y, Ding Z, Zhang S, Wang X. Developing an L-threonineproducing strain from wild-type Escherichia coli by modifying the glucose uptake, glyoxylate shunt, and L-threonine biosynthetic pathway. Biotechnol Appl Biochem. 2019;66:962-76.

70. Wang $P$, Yang $X$, Lin B, Huang J, Tao Y. Cofactor self-sufficient wholecell biocatalysts for the production of 2-phenylethanol. Metab Eng. 2017;44:143-9.

71. Bartek T, Blombach B, Zonnchen E, Makus P, Lang S, Eikmanns BJ, Oldiges M. Importance of NADPH supply for improved L-valine formation in Corynebacterium glutamicum. Biotechnol Prog. 2010;26:361-71.

72. Takeno S, Murata R, Kobayashi R, Mitsuhashi S, Ikeda M. Engineering of Corynebacterium glutamicum with an NADPH-generating glycolytic pathway for L-lysine production. Appl Environ Microbiol. 2010;76:7154-60.

73. Stephanopoulos GN, Aristidou AA, Nielsen J. Chapter 2-Review of cellular metabolism. San Diego: Academic Press; 1998. p. 21-79.

74. Lim SJ, Jung YM, Shin HD, Lee YH. Amplification of the NADPH-related genes $z W f$ and gnd for the oddball biosynthesis of PHB in an E. coli transformant harboring a cloned phbCAB operon. J Biosci Bioeng. 2002:93:543-9.

75. Lee JN, Shin HD, Lee YH. Metabolic engineering of pentose phosphate pathway in Ralstonia eutropha for enhanced biosynthesis of poly- $\beta$ hydroxybutyrate. Biotechnol Prog. 2003;19:1444-9.

76. Poulsen BR, Nøhr J, Douthwaite S, Hansen LV, Iversen JJL, Visser J, Ruijter GJG. Increased NADPH concentration obtained by metabolic engineering of the pentose phosphate pathway in Aspergillus niger. FEBS J. 2005;272:1313-25

77. Li Y, Zhang D, Cai N, Han C, Mao Q, Wang T, Zhou Q, Chen N, Xie X. Betaine supplementation improved L-threonine fermentation of Escherichia coli THRD by upregulating zwf (glucose-6-phosphate dehydrogenase) expression. Electron J Biotechnol. 2019;39:67-73.

78. Fabrizio C, Hess TA, Sylvia H, Wang T, Thomas S, Uwe S. Metabolic flux response to phosphoglucose isomerase knock-out in Escherichia coli and impact of overexpression of the soluble transhydrogenase UdhA. FEMS Microbiol Lett. 2001:204(2):247-52.

79. Lee HC, Kim JS, Jang W, Kim SY. High NADPH/NADP(+) ratio improves thymidine production by a metabolically engineered Escherichia coli strain. J Bacteriol. 2010;149:24-32.

80. Siedler S, Bringer S, Bott M. Increased NADPH availability in Escherichia coli: improvement of the product per glucose ratio in reductive wholecell biotransformation. Appl Microbiol Biotechnol. 2011;92:929-37.

\section{Publisher's Note}

Springer Nature remains neutral with regard to jurisdictional claims in published maps and institutional affiliations. 